\title{
On the spectrum of superspheres
}

\author{
Alessandra Cagnazzo, Volker Schomerus and Vaclav Tlapak \\ DESY Hamburg, Theory Group, \\ Notkestrasse 85, 22607 Hamburg, Germany \\ E-mail: alessandra.cagnazzo@desy.de, volker.schomerus@desy.de, \\ vaclav.tlapak@desy.de
}

ABStRaCT: Sigma models on coset superspaces, such as odd dimensional superspheres, play an important role in physics and in particular the AdS/CFT correspondence. In this work we apply recent general results on the spectrum of coset space models and on supergroup WZNW models to study the conformal sigma model with target space $S^{3 \mid 2}$. We construct its vertex operators and provide explicit formulas for their anomalous dimensions, at least to leading order in the sigma model coupling. The results are used to revisit a nonperturbative duality between the supersphere and the $\operatorname{OSP}(4 \mid 2)$ Gross-Neveu model that was conjectured by Candu and Saleur. With the help of powerful all-loop results for $\frac{1}{2} \mathrm{BPS}$ operators in the Gross-Neveu model we are able to recover the entire zero mode spectrum of the sigma model at a certain finite value of the Gross-Neveu coupling. In addition, we argue that the sigma model constraints and equations of motion are implemented correctly in the dual Gross-Neveu description. On the other hand, high(er) gradient operators of the sigma model are not all accounted for. It is possible that this discrepancy is related to an instability from high gradient operators that has previously been observed in the context of Anderson localization.

KeYwords: Supersymmetry and Duality, Superspaces, Conformal and W Symmetry, Sigma Models

ARXIV EPRINT: 1408.6838 


\section{Contents}

1 Introduction 1

2 The spectrum of coset sigma models 3

2.1 Prologue: vertex operators for flat targets 4

2.2 Vertex operators for $G / H$

2.3 Homogeneous vector bundles on $G / H \quad 6$

2.4 One-loop anomalous dimensions 8

3 The spectrum of the supersphere $S^{3 \mid 2} \quad 10$

$\begin{array}{lll}3.1 & \text { The supersphere sigma model } & 10\end{array}$

$\begin{array}{ll}3.2 & \text { Vertex operators and anomalous dimensions } \\ 3.32\end{array}$

$\begin{array}{lll}3.3 & \text { An alternative construction of vertex operators } & 15\end{array}$

4 Duality with the $\mathfrak{o s p}(4 \mid 2)$ Gross-Neveu model $\quad 16$

$\begin{array}{lll}4.1 & \text { The } \mathfrak{o s p}(4 \mid 2) \text { Gross-Neveu model } & 17\end{array}$

$\begin{array}{lll}4.2 & \text { An all-loop result for deformed WZNW models } & 18\end{array}$

$\begin{array}{ll}4.3 & \text { Checking the proposed duality } 20\end{array}$

$\begin{array}{lll}\text { 4.3.1 Ground state spectrum } & 21\end{array}$

$\begin{array}{lll}\text { 4.3.2 } & \text { Spectrum of gradient operators } & 23\end{array}$

5 Conclusions $\quad 24$

$\begin{array}{ll}\text { A Representation theory of } \mathfrak{o s p}(4 \mid 2) & 26\end{array}$

B Representation theory of $\mathfrak{o s p}(3 \mid 2) \quad 30$

C Restriction of $\mathfrak{o s p}(4 \mid 2)$ representations to $\mathfrak{o s p}(3 \mid 2)$

\section{Introduction}

Non-linear sigma-models (NLSM) play an important role in physics and mathematics. When placed on a 2-dimensional world-sheet, they give rise to renormalizable quantum field theories [1-3]. Initially, 2d NLSMs were mostly studied as toy models of $4 \mathrm{~d}$ gauge theories, for example in order to learn about non-perturbative features and the effect of $\theta$-terms etc., see e.g. [4]. But over the last decades, numerous direct applications were discovered. In string theory, for example, sigma-models on a $2 \mathrm{~d}$ world-sheet are the central ingredient of the perturbative definition.

The properties of sigma models depend on the choice of the target space $\mathcal{M}$ and hence on the particular problem that is addressed. Homogeneous target spaces are particularly 
relevant. In these cases, the target (super)manifold $\mathcal{M}$ admits the transitive action of a continuous Lie (super)group $G$. Consequently, $\mathcal{M}$ can be represented as the coset space $\mathcal{M}=G / H$ where $H$ is the stabilizing (super)subgroup $H \subset G$ of a point on $\mathcal{M}$. Homogeneous (super)spaces $G / H$ for which one can find an automorphism $\gamma: G \rightarrow G$ of order two that leaves all elements in $H \subset G$ fixed are referred to as symmetric. Supercosets $G / H$ in which the subgroup $H$ is fixed by an automorphism of order four play an important role in the AdS/CFT correspondence, see [5] and references therein. While we believe that most of the ideas we are going to develop below apply to a wide class of sigma models on such generalized symmetric superspaces, our presentation and analysis will focus on the coset superspace $\operatorname{OSP}(4 \mid 2) / \operatorname{OSP}(3 \mid 2)$ for which the analysis can be made very explicit.

Understanding sigma models at strong coupling, or equivalently for strongly curved target spaces, is of central importance. In the context of the AdS/CFT correspondence, for example, the strongly curved regime of the string theory is where the dual gauge theory becomes weakly coupled. So, if we had direct control over properties of sigma models on $\mathrm{AdS}_{d+1}$ targets at strong coupling, it would be possible to compare with perturbative gauge theory. Such a comparison could provide deep new insights into the very working of gauge/string dualities, even without the support from supersymmetry and/or integrability. On the other hand, while we proceed to smaller values of the radius $R$, the original background geometry dissolves and we enter a regime that is difficult to analyse.

Nevertheless, there are a few cases in which we indeed understand sigma models for small values of the radius. The first one that comes to mind is the free boson compactified to a circle of radius $R$ which possesses a description involving free fermions when $R=1$. This can be understood through bosonization and is known as Coleman-Mandelstam duality $[6,7]$. Sigma models on complete intersection Calabi-Yau target spaces provide a more intricate family of examples. For many of these models one can find so-called Gepner points, i.e. values of the sigma model coupling at which the theory possesses an exactly solvable description through certain Wess-Zumino-Novikov-Witten (WZNW) models. This duality has been understood most systematically through the use of linear sigma models, see [8].

In this work we present a case study for the sigma model on the supersphere $S^{3 \mid 2}$. With this choice of the target supermanifold, the sigma model coupling turns out to possess vanishing $\beta$ function so that we obtain a continuous family of 2 -dimensional conformal field theories with Virasoro central charge $c=1$. We parametrize the coupling through the radius $R$ of the bosonic base $S^{3}$. We shall discuss the construction of vertex operators and the computation of anomalous dimensions to leading order in $1 / R$ in great detail below, thereby exemplifying constructions and results from [9]. A dual description of this supersphere sigma model has been proposed a few years ago by Candu and Saleur $[10,11]$. It involves a Gross-Neveu-like deformation of a free field theory whose fundamental field multiplet transforms in the fundamental representation of $\operatorname{OSP}(4 \mid 2)$, with four components being fermionic and two bosonic. The free field theory corresponds to the value $R=1$ of the radius in the supersphere sigma model. Extensive tests, mostly based on numerical studies of a lattice discretization, have been performed to support this proposal [10, 11], see also [12].

The OSP (4|2) Gross-Neveu model admits an interpretation as a current-current deformation of an OSP(4|2) Wess-Zumino-Novikov-Witten (WZNW) model at level $k=1$. 
Hence, we can exploit all-loop results from [13] on anomalous dimensions of a certain subset of fields in special truly marginal perturbations of WZNW models. The fields in question are $\frac{1}{2}$ BPS with respect to the target space symmetry, or, in more mathematical terms, they transform in maximally atypical representations. We can apply these results to $\frac{1}{2} \mathrm{BPS}$ fields in the OSP (4|2) Gross-Neveu model. For some special value of the deformation parameter, we are able to identify the low lying spectrum of the supersphere sigma model. The identification includes the one-loop corrections to the conformal dimensions of the supersphere sigma model. For sigma model fields with more than two derivatives the match between the two models is not as convincing and we will uncover a few discrepancies. These would have the potential to disprove the duality conjecture of Candu and Saleur if it were not for some features of the perturbative results on anomalous dimensions that seem to restrict their applicability. The issue will not be settled in this work and merits a deeper investigation.

The relation between a WZNW model at small level and a superspace sigma model we are about to describe illustrates several features that were anticipated by Polyakov in [14]. In particular, we shall see how singular vectors of the WZNW model are related to the sigma model constraint and equation of motion etc. The idea to use precision data on deformed WZNW models in order to test non-perturbative dualities has been put forward previously, mostly in the context of boundary spectra, see $[12,15,16]$. Our work is the first one in which it is applied to bulk spectra. This is made possible mostly by the technical advances in $[9,13]$. Let us also point out that conformal sigma models are not that rare when the target space is a supermanifold, see e.g. [17-22] and references therein. In this sense, the ideas we put forward below should apply to a much wider class of examples.

The plan of the paper is as follows. In the next section we shall review the results of [9] on the construction of vertex operators and their one-loop anomalous dimensions in coset space sigma models. These are then worked out explicitly for the supersphere model with target space $S^{3 \mid 2}$. For vertex operators involving a small number of derivatives we compare our general prescriptions with more conventional constructions of vertex operators in terms of the fundamental field multiplet. The comparison illustrates how advantageous the new approach is in enumerating physical fields, though once the dust settles both approaches certainly give the same results. In section 4 we then turn to the proposed dual Gross-Neveu model, describe its field content and the deformation away from the free field theory. After a brief review of results from [13] we analyse the low lying $\frac{1}{2} \mathrm{BPS}$ spectrum for the value of the Gross-Neveu coupling that is conjectured to correspond to the weakly coupled sigma model. We shall find intriguing agreements, but also some discrepancies. These are briefly discussed in the concluding section along with a number of interesting open problems.

\section{The spectrum of coset sigma models}

The aim of this section is to review some results from [9] concerning the spectrum of sigma models on symmetric superspaces. After a bit of introduction we shall build a basis of fields in sigma models on coset (super-)spaces $G / H$. At least when $G / H$ is symmetric our basis diagonalizes the one-loop dilation operator and we can give a very simple formula for the spectrum of one-loop anomalous dimensions. The material of this section has been 
split into three different subsections of decreasing generality. While the construction of field operators in the first subsection holds for all coset models $G / H$, our discussion of the zero modes is limited to compact $G$. Results on the one-loop spectrum have only been obtained for symmetric (super-)spaces, though an extension to generalized symmetric spaces is under investigation.

\subsection{Prologue: vertex operators for flat targets}

Before we can review what is known about the spectrum of weights we need to recall the construction of vertex operators from [9]. Let us motivate the prescription given there with a few comments on the usual vertex operators of a free boson, i.e. a sigma model on the coset space $S^{1}=\mathrm{SO}(2) / \mathrm{SO}(1)$ with trivial denominator group $H=\mathrm{SO}(1)=\{e\}$. As is well known, the space of such operators is spanned by

$$
\Phi_{k ; \mathbf{p}, \overline{\mathbf{p}}}(z, \bar{z})=e^{i k \theta(z, \bar{z})} \mathbf{p}_{\mathbf{m}}(j, \partial j, \ldots) \overline{\mathbf{p}}_{\overline{\mathbf{m}}}(\bar{\jmath}, \bar{\partial} \bar{\jmath}, \ldots) .
$$

Here, $j=j(z)$ is the current $j=i \partial \theta$ and $\bar{\jmath}$ is of the same form but with a derivative $\bar{\partial}$ instead of $\partial$, i.e. $\bar{\jmath}=i \bar{\partial} \theta$. The object $\mathbf{p}_{\mathbf{m}}$ denotes the monomial

$$
\mathbf{p}_{\mathbf{m}}(j, \partial j, \ldots)=j^{m_{1}}(\partial j)^{m_{2}} \ldots
$$

in $j$ and its derivatives. The powers $m_{i}$ are components of the multi-index $\mathbf{m}=\left(m_{1}, m_{2}, \ldots\right)$ we have placed on $\mathbf{p}$. Of course, the definition of $\overline{\mathbf{p}}$ is similar, but with derivatives $\bar{\partial}$ instead of $\partial$. Note that the multi-index $\overline{\mathbf{m}}$ is independent of $\mathbf{m}$.

The operators $\exp (i k \theta)$ are associated to the zero modes of the free boson, i.e. there is one such operator for each function on the target space. For $\mathbf{m}=0=\overline{\mathbf{m}}$ we obtain the usual tachyon vertex operators. The choice $\mathbf{m}=(1,0,0, \ldots)=\overline{\mathbf{m}}$ corresponds to the vertex operators for massless states etc.

\subsection{Vertex operators for $G / H$}

In generalizing this discussion to non-trivial coset models $G / H$ we must address how to replace the currents $j$ and $\bar{\jmath}$, the tail monomials $\mathbf{p}$ and $\overline{\mathbf{p}}$ and the zero mode contributions $\exp (i k X)$.

Let us begin with the fields $j$ and $\bar{\jmath}$. One could imagine to simply take derivatives of coordinate fields $\theta_{J}$ that are associated with some choice of coordinates on $G / H$. While this works just fine for a flat target space, it is not the smartest choice for curved backgrounds. Instead, we shall adopt the definition

$$
j_{\alpha}:=E_{\alpha}^{J}(\theta) \partial \theta_{J}, \quad \bar{\jmath}_{\alpha}:=E_{\alpha}^{J}(\theta) \bar{\partial} \theta_{J}
$$

where $E_{\alpha}^{J}$ is the vielbein for our coset space. Equivalently, if we think of the points on $G / H$ as being parametrized by orbits of group elements $g \in G$ under the right action of $H$, we can also construct $j$ and $\bar{\jmath}$ as

$$
j_{\alpha}=\left(g^{-1} \partial g, t_{\alpha}\right), \quad \bar{\jmath}_{\alpha}=\left(g^{-1} \bar{\partial} g, t_{\alpha}\right) .
$$


Here, $t_{\alpha}$ runs through a basis in the quotient space $\mathfrak{m}=\mathfrak{g} / \mathfrak{h}$. The space $\mathfrak{m}$ carries an action of the denominator Lie (super-)algebra $\mathfrak{h}$. Its dimension coincides with the dimension of $G / H$. Note that there is one crucial difference with respect to the flat target $S^{1}$, namely our fields $j$ and $\bar{j}$ transform non-trivially under the action of the denominator algebra. Of course, physical fields of the coset model must be invariant. Hence, it will be important to keep track of how the composite fields we are about to construct transform under $\mathfrak{h}$.

A field can contain arbitrary products of $j_{\alpha}$ and $\bar{j}_{\alpha}$ and their derivatives, just as for flat targets. Since the multiplets $\left(j_{\alpha}\right)$ and $\left(\bar{\jmath}_{\alpha}\right)$ transform in the representation $\mathfrak{m}$ of $\mathfrak{h}$, we can build tails in any subrepresentation $[\mu]$ that appears in some tensor power of $\mathfrak{m}$. More precisely, we can pick two multi-indices $\mathbf{m}$ and $\overline{\mathbf{m}}$ as in our discussion of the compactified free boson and then choose two intertwiners

$$
\mathbf{P}_{\mu, \mathbf{m}}: \bigotimes_{i} \mathfrak{m}^{\odot m_{i}} \rightarrow[\mu], \quad \overline{\mathbf{P}}_{\bar{\mu}, \overline{\mathbf{m}}}: \bigotimes_{i} \mathfrak{m}^{\odot \bar{m}_{i}} \rightarrow[\bar{\mu}]
$$

Here, we used $\mathfrak{m}^{\odot m}$ to denote the $\mathrm{m}$-fold (graded) symmetric tensor power of $\mathfrak{m}$. Given any such intertwiner, we construct the tail factor

$$
\mathbf{P}_{\mu, \mathbf{m}}(j, \partial j, \ldots)=\mathbf{P}_{\mu, \mathbf{m}}\left[j^{\otimes^{m_{1}}} \otimes(\partial j)^{\otimes^{m_{2}}} \otimes \cdots\right]
$$

and similarly for the second contribution that involves $\bar{\jmath}$ and its derivatives with respect to $\bar{\partial}$. We have used tensor products and powers instead of ordinary ones to remind us that $j$ is a multi-component object. Note that there is a finite number of intertwiners $\mathbf{P}_{\mu, \mathbf{m}}$ and $\overline{\mathbf{P}}_{\bar{\mu}, \overline{\mathbf{m}}}$ for any given choice of $\mathbf{m}$ and $\overline{\mathbf{m}}$. This finite choice has no analogue in a flat background.

Having discussed the tail of our vertex operators, we also need to address the zero mode factors. In the compactified free boson the zero mode contribution was a function on the target space. Functions on the coset space $G / H$ can be thought of as $H$-invariant functions on the group $G$. But since our tail factors transform non-trivially under $H$, it seems natural to admit zero mode contributions whose transformation behavior under the right action of $H$ on $G$ is non-trivial as well. More precisely, for any given representation $S_{\lambda}$ of $H$ on the carrier space $\mathcal{S}_{\lambda}$ let us consider the following space of $\mathcal{S}_{\lambda}$-valued functions on $G$,

$$
\Gamma_{\lambda}=\Gamma_{\lambda}(G / H)=\left\{F \in L_{2}(G) \otimes \mathcal{S}_{\lambda}: F(g h)=S_{\lambda}\left(h^{-1}\right) F(g) \forall h \in H\right\} .
$$

Elements of the linear space $\Gamma_{\lambda}$ may be considered as sections in a homogeneous vector bundle on $G / H$ [23]. We will analyse the structure of these vector bundles in the next subsection.

At this point we have discussed three ingredients of our vertex operators, namely the tail factors $\mathbf{P}_{\mu, \mathbf{m}}$ and $\overline{\mathbf{P}}_{\bar{\mu}, \overline{\mathbf{m}}}$ along with the zero mode contribution $V \in \Gamma_{\lambda}$. These transform in the representations $\mu, \bar{\mu}$ and $\lambda$ of the denominator algebra $\mathfrak{h}$. Obviously, a physical field in the coset model must be $\mathfrak{h}$ invariant. Hence, we must glue our three ingredients with an intertwiner

$$
\mathbf{C}^{\lambda \mu \bar{\mu}}:[\lambda] \otimes[\mu] \otimes[\bar{\mu}] \rightarrow \mathbb{C}
$$

from the triple tensor product between the representations $[\lambda],[\mu]$ and $[\bar{\mu}]$ of the denominator algebra $\mathfrak{h}$ to the complex numbers. Fields of the coset model now take the form

$$
\Phi_{\Lambda, \lambda, \mu, \bar{\mu}}(z, \bar{z})=V_{\Lambda \lambda}(z, \bar{z}) \mathbf{P}_{\mu ; \mathbf{m}}(j, \partial j, \ldots) \overline{\mathbf{P}}_{\bar{\mu} ; \overline{\mathbf{m}}}(\bar{\jmath}, \bar{\partial} \bar{\jmath}, \ldots) \mathbf{C}^{\lambda \mu \bar{\mu}},
$$


where $V_{\Lambda \lambda} \in \Gamma_{\lambda}$ is a section that transforms in the representation $\Lambda$ of the numerator algebra $\mathfrak{g}$. By construction, these fields are invariant under the action of the denominator group $H$. On the other hand, the action of the numerator group $G$ is non-trivial. It is determined by the way the section $V_{\Lambda \lambda}$ transforms. The label $\Lambda$ is the curved space analogue of the linear momentum $k$ in a circular target $S^{1}$.

The labels $(\Lambda, \lambda, \mu, \bar{\mu})$ we have placed on the symbol $\Phi$ do not keep track of all the freedom we have in the construction of vertex operators. In order to count all possible fields of the coset model one needs to count the intertwiners $\mathbf{P}, \overline{\mathbf{P}}$ and $\mathbf{C}$ that were introduced in eqs. (2.4) and (2.7), respectively. In addition, there is often some freedom in the choice of the section $V_{\Lambda \lambda} \in \Gamma_{\lambda}$. While the number of intertwiners may be determined straightforwardly from the fusion rules of the Lie (super-)algebra $\mathfrak{h}$, the space of sections in homogeneous vector bundles requires input from harmonic analysis. We will analyse the space $\Gamma_{\lambda}$ in the next subsection. For $O(N)$ vector models, i.e. the coset sigma models with target space $O(N) / O(N-1)$, the space of fields has been counted in [9] and the result was shown to agree with other descriptions of the field space for these models.

\subsection{Homogeneous vector bundles on $G / H$}

As we explained in the previous subsection, a good control over vertex operators of coset models requires some knowledge about sections in homogeneous vector bundles over $G / H$ and their transformation behavior under the (left) action of $G$. Our main goal in this subsection is to explain the decomposition

$$
\Gamma_{\lambda} \cong \sum_{\Lambda} n_{\Lambda \lambda}[\Lambda]
$$

Here, the linear space $\Gamma_{\lambda}$ is considered as a representation of the numerator Lie (super-)algebra $\mathfrak{g}$. The summation on the right hand side runs over irreducible representations $[\Lambda]$ of this algebra. Let us stress that for Lie superalgebras, the sum is not direct, at least not in general. We will return to this issue below.

In the expansion (2.9), each summand $[\Lambda]$ appears with some multiplicity $n_{\Lambda \lambda}$. Following standard mathematical notation, we shall also write

$$
n_{\Lambda \lambda}=\left[\Gamma_{\lambda}: \mathcal{S}_{\Lambda}\right]
$$

for the number of times a given irreducible representation $\mathcal{S}_{\Lambda}$ of $\mathfrak{g}$ appears in (the decomposition series of) the space $\Gamma_{\lambda}$ of sections. It is a central result from harmonic analysis of compact supergroups that

$$
\left[\Gamma_{\lambda}: \mathcal{S}_{\Lambda}\right]=\left[\left.\mathcal{P}_{\Lambda}\right|_{\mathfrak{h}}: \mathcal{P}_{\lambda}\right]
$$

The objects $\mathcal{P}_{\Lambda}$ and $\mathcal{P}_{\lambda}$ denote representations of the Lie superalgebras $\mathfrak{g}$ and $\mathfrak{h}$, respectively. These particular representations are called projective covers, see e.g. [24, 25] for a precise definition and more background. They coincide with the irreducible representations $\mathcal{S}_{\Lambda}$ and $\mathcal{S}_{\lambda}$ when no shorting conditions are satisfied, i.e. when both $\Lambda$ and $\lambda$ are non-BPS. The case of BPS (or atypical) multiplets will be discussed in more detail below. After restriction to $\mathfrak{h} \subset \mathfrak{g}$, the representation $\mathcal{P}_{\Lambda}$ gives rise to a representation $\left.\mathcal{P}_{\Lambda}\right|_{\mathfrak{h}}$ of $\mathfrak{h}$. The number on 
the right hand side of equation (2.11) denotes the number of times the representation $\mathcal{P}_{\lambda}$ appears in the representation $\left.\mathcal{P}_{\Lambda}\right|_{\mathfrak{h}}$.

All this might seem a bit abstract at first. So, let us briefly illustrate the content of eq. (2.11) for the coset space $S^{2}=\mathrm{SU}(2) / \mathrm{U}(1)$. In this case, there exists an infinite set of complex line bundles which are parametrized by the monopole number $k \in \mathbb{Z}$. This number and hence the associated bundles are in one-to-one correspondence with irreducible representations $\mathcal{S}_{k}$ of the denominator group $H=\mathrm{U}(1)$. For monopole number $k=0$ we are dealing with the trivial line bundle, i.e. with functions on $S^{2}$. Of course we know very well how the space of functions decomposes under the action of $\mathfrak{s u}(2)$ : each integer spin representation appears with multiplicity one. We may recover this fact from our formula (2.11) as follows. The space of functions on $S^{2}$ is associated to the label $\lambda=0$. We want to know how many times an irreducible representation $\mathcal{S}_{\Lambda}=\mathcal{S}_{j}$ of $\mathfrak{s u}(2)$ appears in the decomposition of $\Gamma_{0}$. According to eq. (2.11), this number is given by

$$
\left[\Gamma_{0}: \mathcal{S}_{j}\right]=\left[\left.\mathcal{S}_{j}\right|_{\mathrm{U}(1)}: \mathcal{S}_{0}\right]=\left\{\begin{array}{l}
1 \text { for } j \in \mathbb{N} \\
0 \text { for } j \in \mathbb{N}+\frac{1}{2} .
\end{array}\right.
$$

Here $\mathcal{S}_{0}$ denotes the trivial representation of $\mathfrak{h}$. For bosonic Lie groups, we do not have to distinguish between projective covers $\mathcal{P}_{j}$ and irreducibles, i.e. $\mathcal{S}_{j}=\mathcal{P}_{j}$. The second equality follows from the fact that the spin $j$ representation $\mathcal{S}_{j}$ contains exactly one state on which the generator $J^{3}$ of the $\mathfrak{u}(1) \subset \mathfrak{s u}(2)$ has zero eigenvalue if and only if $j$ is integer. For non-trivial monopole line bundles, the evaluation proceeds along the same lines. In this case the space $\Gamma_{k}$ of sections contains each integer spin representation $\mathcal{S}_{j}$ satisfying $j \geq k$ with multiplicity one.

The only additional complication we have to deal with in applying eq. (2.11) to superspaces comes from the distinction between irreducibles and projective covers. For typical (long) multiplets $\mathcal{S}_{\Lambda}$ of a Lie superalgebra $\mathfrak{g}$, the projective cover $\mathcal{P}_{\Lambda}$ agrees with $\mathcal{S}_{\Lambda}=\mathcal{P}_{\Lambda}$. But if $\mathcal{S}_{\Lambda}$ is an atypical (short) multiplet then $\mathcal{P}_{\Lambda} \neq \mathcal{S}_{\Lambda}$ is an indecomposable representation. It should be considered as a very specific 'composite' representation that is built from several short multiplets. For the Lie superalgebra $\mathfrak{g}=\mathfrak{o s p}(4 \mid 2)$ the projective covers are discussed explicitly in appendix A. Of course, short representations of the denominator algebra $\mathfrak{h}$ can also be combined into projective covers, see appendix $\mathrm{B}$ where the projective covers for $\mathfrak{o s p}(3 \mid 2)$ are discussed. Let us finally mention that upon restriction from $\mathfrak{g}$ to the subalgebra $\mathfrak{h} \subset \mathfrak{g}$, a projective cover $\mathcal{P}_{\Lambda}$ decomposes into a direct sum of projective covers $\mathcal{P}_{\lambda}$. Hence, the numbers on the right hand side of eq. (2.11) are well defined. We shall compute them for homogeneous vector bundles on the supersphere $S^{3 \mid 2}$ later on.

Let us briefly mention one simple example that can be used to illustrate how important the distinction between irreducibles and projective covers is. To this end we consider the homogeneous vector bundle $\Gamma_{\mathrm{ad}}$ on the supersphere $S^{3 \mid 2}$ that is associated with the adjoint representation of the denominator algebra $\mathfrak{o s p}(3 \mid 2)$. It turns out that this bundle contains two multiplets of sections which transform in the adjoint representation $\mathcal{S}_{\mathrm{Ad}}$ of the numerator algebra osp $(4 \mid 2)$, i.e. $\left[\Gamma_{\mathrm{ad}}: \mathcal{S}_{\mathrm{Ad}}\right]=2$. On the other hand, the adjoint representation of $\mathfrak{o s p}(4 \mid 2)$ is 17-dimensional and that of $\mathfrak{o s p}(3 \mid 2)$ is 12-dimensional. Hence, 
for dimensional reasons, the restriction of $\mathcal{S}_{\text {Ad }}$ to $\mathfrak{o s p}(3 \mid 2)$ contains $\mathcal{S}_{\text {ad }}$ only once,

$$
2=\left[\left.\mathcal{P}_{\text {Ad }}\right|_{\mathfrak{h}}, \mathcal{P}_{\text {ad }}\right] \neq\left[\left.\mathcal{S}_{\text {Ad }}\right|_{\mathfrak{h}}, \mathcal{S}_{\text {ad }}\right]=1
$$

This example demonstrates that harmonic analysis on superspaces requires a bit of extra care precisely because of the existence of BPS representations.

Before we conclude this subsection let us stress once more that formula (2.11) is restricted to compact (super-)algebras. This does not mean that similar control of homogeneous vector bundles can not be achieved when $G$ is non-compact. As long as $H$ is compact, one can continue to derive results on the decomposition of homogeneous vector bundles from the harmonic analysis of $G$. So, if the latter is understood, homogeneous vector bundles pose no additional problems. When $H$ in non-compact, however, normalizable sections of on $G / H$ are no longer obtained from normalizable functions on $G$ and hence cosets with non-compact denominator require an independent analysis. Nevertheless, the decomposition of homogeneous vector bundles is known in many concrete examples.

\subsection{One-loop anomalous dimensions}

While our construction of vertex operators in coset sigma models was completely general and the property (2.11) holds for all homogeneous vector bundles on quotients $G / H$ of a compact Lie (super-)group $G$, the following results on the one-loop corrections to the spectrum of coset models have only been derived for symmetric (super-)spaces, although work on so-called generalized symmetric spaces, including those relevant for the AdS/CFT correspondence, is in progress.

The computations carried out in [9] show that the one-loop anomalous dimensions depend only on the representation labels $\Lambda, \lambda, \mu, \bar{\mu}$ and not on the intertwiners $\mathbf{P}, \overline{\mathbf{P}}$ and $\mathbf{C}$ that enter the construction of fields (2.8) in the coset model. This is why we labeled our fields $\Phi$ by a subscript that makes no reference to the precise choice of intertwiners.

At zero sigma model coupling, i.e. for $R=\infty$, the sigma model fields possess their naive dimensions $\left(h_{\infty}, \bar{h}_{\infty}\right)$ that are given by the number of derivatives,

$$
h_{\infty}=\sum_{j=1}^{\infty} j m_{j}, \quad \bar{h}_{\infty}=\sum_{j=1}^{\infty} j \bar{m}_{j},
$$

where only finitely many of the $m_{j}$ are non-zero. Once we turn on the interaction, these scaling weights are shifted by the so called anomalous dimension $\delta_{R} h$, i.e. at some finite value of the coupling $R$ the scaling weights have the form

$$
(h(R), \bar{h}(R))=\left(h_{\infty}+\delta_{R} h, \bar{h}_{\infty}+\delta_{R} h\right) .
$$

According to [9], the leading contribution to the anomalous dimension takes the form

$$
\delta_{R}^{(1)} h=\frac{1}{2 R^{2}}\left(\operatorname{Cas}_{\mathfrak{g}}(\Lambda)-\operatorname{Cas}_{\mathfrak{h}}(\mu)-\operatorname{Cas}_{\mathfrak{h}}(\bar{\mu})\right) .
$$

In the derivation the result actually emerges as a sum of two different pieces that are associated with the zero mode factor and the tail of the vertex operator, respectively. 
Recall that the zero mode factor $V_{\Lambda \lambda}$ is a section in a homogeneous vector bundle $\Gamma_{\lambda}$. Such sections are acted upon by the Lichnerowicz Laplacian $\Delta_{L}$, whose eigenvalues were expressed through the quadratic Casimir operators of $\mathfrak{g}$ and $\mathfrak{h}$ in [26],

$$
\Delta_{L} V_{\Lambda \lambda}(\theta)=\left(\operatorname{Cas}_{\mathfrak{g}}(\Lambda)-\operatorname{Cas}_{\mathfrak{h}}(\lambda)\right) V_{\Lambda \lambda}(\theta) .
$$

The contribution of the tail factors to the annomalous dimension can be written as a spinspin interaction between fields $j$ and $\bar{\jmath}$. It leads to a term of the form $\operatorname{Cas}_{\mathfrak{h}}(\lambda)-\operatorname{Cas}_{\mathfrak{h}}(\mu)-$ $\operatorname{Cas}_{\mathfrak{h}}(\bar{\mu})$. Note that the first term in this combination cancels the constant shift $\operatorname{Cas}_{\mathfrak{h}}(\lambda)$ in the eigenvalues of the Lichnerowicz Laplacian so that we end up with the expression given in eq. (2.16).

Formula (2.16) is actually very general. It holds for all sigma models on symmetric superspaces with vanishing beta function. When properly interpreted, see [9], it can also be used for models with world-sheet supersymmetry, such as e.g. the $N=2$ worldsheet supersymmetric sigma model on complex projective superspace $\mathbb{C P}^{3 \mid 4}$ etc. In applications to non-conformal theories, such as e.g. the usual $\mathrm{O}(N)$ models, the formula for $\delta^{(1)} h$ requires a simple additional term,

$$
\delta_{R}^{(1)} h=\frac{1}{2 R^{2}}\left(\operatorname{Cas}_{\mathfrak{g}}(\Lambda)-\mathbf{C a s}_{\mathfrak{h}}(\mu)-\mathbf{C a s}_{\mathfrak{h}}(\bar{\mu})+\mathbf{C a s}_{\mathfrak{h}}(\mathfrak{m}) \sum_{i}\left(m_{i}+\bar{m}_{i}\right)\right) .
$$

Since vanishing of the one-loop beta function requires that $\mathbf{C a s}_{\mathfrak{h}}(\mathfrak{m})=0$ we recover the formula (2.16) for conformal sigma models. Our simple formula (2.16) or rather its generalization (2.18) summarizes and extends the results of many papers in which anomalous dimensions, mostly dealing with $\mathfrak{g}$-invariant fields, have been studied model by model, see e.g. [27-33]. That all these computations may be captured by a single universal formula (2.18) is quite remarkable. Of course, this success is intimately tied to the construction (2.8) of vertex operators. We now see how well this construction was adapted to the computation of 1-loop anomalous dimensions.

Much of the previous work on anomalous dimensions of (high-)gradient operators in sigma models was motivated by a somewhat puzzling instability that has first been observed in $\mathrm{O}(N)$ vector models [27] and later understood to be a rather generic feature of sigma model perturbation theory, see [34] and references therein. This instability arises because of the negative sign in front of the terms $\mathbf{C a s}_{\mathfrak{h}}(\mu)$ and $\mathbf{C a s}_{\mathfrak{h}}(\bar{\mu})$. Naively one might think that high gradient operators, i.e. operators (2.8) for which $\sum_{j} j\left(m_{j}+\bar{m}_{j}\right)=h_{\infty}+\bar{h}_{\infty}$ is large, are highly irrelevant. But it turns out that some of these operators acquire a very large negative anomalous dimension. More precisely, one can show that for every choice of the sigma model coupling $R^{-2}$, no matter how small, one can find a $\mathfrak{g}$-invariant high gradient operator $\mathcal{O}=\Phi_{0, \lambda, \mu, \bar{\mu}}$ such that

$$
h_{\infty}(\mathcal{O})+\bar{h}_{\infty}(\mathcal{O})-\frac{1}{R^{2}}\left(\operatorname{Cas}_{\mathfrak{h}}(\mu)+\operatorname{Cas}_{\mathfrak{h}}(\bar{\mu})\right)<2 .
$$

This is because $\operatorname{Cas}_{\mathfrak{h}}(\mu)$ grows quadratically with the weights of the representation $\mu$ and the maximal weight grows linearly with the number of currents $j$ in the tail. On the other 
hand, the positive contribution $h_{\infty}(\mathcal{O})$ only grows linearly in the number of $j$ s. The argument shows that (infinitely many) high gradient operators become relevant for arbitrarily small sigma model coupling. One could have hoped that higher orders in perturbation theory correct the issue, but they turn out to make things even worse [35]. We would be ready to conclude that sigma models are inherently unstable if it were not for the many independent studies, e.g. through lattice discretizations, that display no pathologies. As far as we know, the problem has never been resolved but it is something to be kept in mind as we proceed.

\section{The spectrum of the supersphere $S^{3 \mid 2}$}

The aim of this section is twofold. Partly, we would like to illustrate the general results we have reviewed in the previous section through the simplest nontrivial example of an interacting conformal sigma model, namely the theory with target space $S^{3 \mid 2}$. This supersphere can be considered as a quotient $G / H$ of the compact supergroup $G=\operatorname{OSP}(4 \mid 2)$ by the subgroup $H=\operatorname{OSP}(3 \mid 2)$. Since the latter is fixed by an order two automorphism

of the former, the supersphere $S^{3 \mid 2}$ is a compact symmetric superspace. Hence, all the results we outlined in the previous section apply to this case. Our task is to work them out explicitly. This will require some input from the representation theory of $\mathfrak{o s p}(4 \mid 2)$ and $\mathfrak{o} \mathfrak{s p}(3 \mid 2)$ which can be found in several appendices. The second purpose of this section is to gather some data about the supersphere sigma model that we can later use to test the conjectured duality with the OSP(4|2) Gross-Neveu model.

We will begin by describing several equivalent formulations of the supersphere sigma model. Concrete results on low gradient operators and their anomalous dimensions are worked out in the second subsection. In the third subsection we describe the more conventional construction of (low gradient) vertex operators in terms of the fundamental field of the non-linear sigma model. While this turns out to be significantly more cumbersome than the approach advocated in the previous subsection, it will allow us to understand the impact of symmetries and equations of motion.

\subsection{The supersphere sigma model}

The most basic description of the supersphere $S^{3 \mid 2}$ is as a co-dimension one supermanifold in the flat superspace $\mathbb{R}^{4 \mid 2}$ defined by the equation

$$
X \cdot X:=\sum_{j=1}^{4} x_{j}^{2}+2 \eta_{1} \eta_{2}=1
$$

Here, $x_{j}, j=1, \ldots, 4$, and $\eta_{1}, \eta_{2}$, are the bosonic and fermionic coordinates of $\mathbb{R}^{4 \mid 2}$, respectively. We shall often combine these coordinates into a multiplet of supercoordinates $X=\left(X_{A}\right)=\left(x_{j}, \eta_{1}, \eta_{2}\right)$. For a pair $X$ and $Y$ in such multiplets the inner product $\cdot$ is defined as

$$
X \cdot Y=\sum_{j} x_{j} y_{j}+\eta_{1} \xi_{2}-\eta_{2} \xi_{1}
$$


Here, we have denoted the fermionic coordinates of $Y$ by $\xi_{1}$ and $\xi_{2}$. We can now write the action of the associated sigma model as

$$
\mathcal{S}^{\mathrm{SM}}[X, \rho]=\frac{R^{2}}{2 \pi} \int d^{2} z(\partial X \cdot \partial X-\rho(X \cdot X-1)) .
$$

Here $\rho$ is a Lagrange multiplier that implements the supersphere constraint (3.1). The parameter $R$ can be interpreted as the radius of the supersphere. In the regime where $R$ is large, the sigma model is weakly coupled and perturbation theory should give reliable results. The equations of motion for the field multiplet $X$ read

$$
\partial \bar{\partial} X=(\partial X \cdot \bar{\partial} X) X .
$$

From our description of the supersphere through equation (3.1) it is evident that $S^{3 / 2}$ comes equipped with an $\mathfrak{o s p}(4 \mid 2)$ action. In fact, the Lie superalgebra $\mathfrak{o s p}(4 \mid 2)$ acts on the embedding space $\mathbb{R}^{4 \mid 2}$ through its fundamental representation. By the very definition of $\mathfrak{o s p}(4 \mid 2)$ this action respects the constraint (3.1). The supersphere $S^{3 \mid 2}$ can be obtained from the supergroup $\operatorname{OSP}(4 \mid 2)$ by taking the following quotient

$$
S^{3 \mid 2}=\operatorname{OSP}(4 \mid 2) / \operatorname{OSP}(3 \mid 2)
$$

with respect to the right action of the subsupergroup $\operatorname{OSP}(3 \mid 2) \subset \operatorname{OSP}(4 \mid 2)$. The latter appears as the stabilizer of a point $X=\left(X_{A}\right)=(1,0,0, \ldots)$ on the supersphere. Since this stabilizer is left invariant by the reflection of the first coordinate, the quotient (3.5) is a symmetric superspace. In conclusion, we have shown that the sigma model (3.3) possesses all the properties that we assumed in the previous section.

In order to get a better feeling for how non-trivial the supersphere sigma model really is, we solve the constraint (3.1) explicitly. To this end, we parametrize $S^{3 \mid 2}$ through three angular coordinates $\vartheta_{j}$ and 2 fermionic variables $\eta_{b}$. The line element takes the following form

$$
d s^{2}=2\left(1-\eta_{1} \eta_{2}\right) d \eta_{1} d \eta_{2}+\left(1-2 \eta_{1} \eta_{2}\right) d \Omega_{3}
$$

where

$$
d \Omega_{3}=d \vartheta_{1}^{2}+\cos ^{2} \vartheta_{1} d \vartheta_{2}^{2}+\sin ^{2} \vartheta_{1} d \vartheta_{3}^{2}
$$

is the usual line element of the 3-dimensional unit sphere. In the sigma model, the coordinates are promoted to fields and the action reads

$$
\begin{aligned}
\mathcal{S}^{\mathrm{SM}}[\vartheta, \eta]=\frac{R^{2}}{2 \pi} \int d^{2} z & \left(2\left(1-\eta_{1} \eta_{2}\right)\left(\partial \eta_{1} \bar{\partial} \eta_{2}-\partial \eta_{2} \bar{\partial} \eta_{1}\right)\right. \\
+ & \left.\left(1-2 \eta_{1} \eta_{2}\right)\left(\partial \vartheta_{1} \bar{\partial} \vartheta_{1}+\cos ^{2} \vartheta_{1} \partial \vartheta_{2} \bar{\partial} \vartheta_{2}+\sin ^{2} \vartheta_{1} \partial \vartheta_{3} \bar{\partial} \vartheta_{3}\right)\right)
\end{aligned}
$$

For the sigma model on the purely bosonic 3 -sphere the coupling $R$ runs and in order for the flow to end in a non-trivial fixed-point one must add a Wess-Zumino term [36]. But the presence of the two fermionic directions changes the situation profoundly. As shown in [19], the $\beta$-function of the sigma model on $S^{3 \mid 2}$ is the same as for a bosonic sigma model on a sphere $S^{d}$ whose dimension $d=3-2=1$ is given by the difference between the 
number of bosonic and fermionic coordinates. Consequently, the $\beta$-function vanishes for the sigma model on $S^{3 \mid 2}$, i.e. the model (3.7), defines a family of non-unitary interacting conformal field theories at central charge $c=1$ with continuously varying exponents.

Before we apply the results reviewed in the previous section to this model let us note that the action (3.7) can be written very compactly if we factorize the metric with the help of the super-Vielbeins $E_{\alpha}^{J}(\vartheta, \eta)$,

$$
g^{I J}(\vartheta, \eta):=\kappa^{\alpha \beta} E_{\alpha}^{I}(\vartheta, \eta) E_{\beta}^{J}(\vartheta, \eta)(-1)^{|\beta|(|I|+|\alpha|)}
$$

where $\kappa$ is the invariant form of $\mathfrak{o s p}(4 \mid 2)$ and the indices $\alpha, \beta$ run over directions along the quotient $\mathfrak{m}=\mathfrak{o s p}(4 \mid 2) / \mathfrak{o s p}(3 \mid 2)$. We can now combine the Vielbeins with the derivatives of the coordinate fields $\left(\theta_{J}\right)=\left(\vartheta_{j}, \eta_{a}\right)$ as in eq. (2.2) to obtain

$$
\mathcal{S}^{\mathrm{SM}}[\theta]=\frac{R^{2}}{2 \pi} \int d^{2} z g^{I J}(\vartheta, \eta) \partial \theta_{I} \bar{\partial} \theta_{J}=\frac{R^{2}}{2 \pi} \int d^{2} z \kappa^{\alpha \beta} j_{\alpha}(z, \bar{z}) \bar{\jmath}_{\beta}(z, \bar{z}) .
$$

Of course, all the non-linearity of the action (3.7) is just hidden in the complicated structure of the fields $j$ and $\bar{\jmath}$. Note that the latter transform in the fundamental representation of the stabilizer subgroup $\operatorname{OSP}(3 \mid 2)$. In the action the corresponding index $\alpha$ is contracted with the $\beta$ so as to give an invariant.

Unlike the sigma model on $S^{1}=\mathrm{U}(1)$, the theory defined by the action (3.7) is not free. For large radius $R$, the model is weakly coupled and its properties may by studied perturbatively. But as we pass to a more strongly curved background, computing quantities as a function of the radius $R$ may seem like a very daunting task. This is even more so because there is very little symmetry to work with. As a conformal field theory, the sigma model on the supersphere possesses the usual chiral Virasoro symmetries. But for a model with multiple bosonic coordinates the two sets of chiral Virasoro generators are not sufficient to make the theory rational. Since there are no efficient tools to construct the theory at generic values of the radius parameter $R$, finding a dual description whose perturbative regime describes a strongly curved supersphere is of obvious interest.

\subsection{Vertex operators and anomalous dimensions}

Before we can begin constructing vertex operators for the supersphere sigma model we need a little bit of background on representations of both $\mathfrak{o s p}(4 \mid 2)$ and $\mathfrak{o s p}(3 \mid 2)$. A much more comprehensive discussion can be found in the appendices. It is heavily based on two papers by van der Jeugt [37, 38].

The Lie superalgebra osp $(4 \mid 2)$ possesses the bosonic subalgebra $\mathfrak{s o}(4) \oplus \mathfrak{s p}(2)$. Since this has rank $r=3$, generic representations are labeled by triples of weights $\left[j_{1}, j_{2}, j_{3}\right]$. Atypical (or BPS) representations satisfy a single shortening condition. The possible conditions are listed in eq. (A.3). With one such condition relating the three weights $j_{i}$, atypical representations $\Lambda_{l, k}$ are labeled by two integers $l \geq 0$ and $k$. The precise relation between $l, k$ and the weights $j_{i}$ are given in eqs. (A.5) and (A.6). Let us only note that the label of the trivial representation is $\Lambda_{0,0}$ while that of the 17 -dimensional adjoint is $\Lambda_{0,1}$. The representations $\Lambda_{l, 0}$ on the other hand are associated with (graded) symmetric traceless tensors of $\mathfrak{o s p}(4 \mid 2)$. 
In the atypical representation $\Lambda_{l, k}$, the quadratic Casimir element $\mathbf{C a s}_{\mathfrak{g}}$ takes the value

$$
\operatorname{Cas}_{\mathfrak{g}}\left(\Lambda_{l, k}\right)=l^{2} .
$$

We conclude that the Casimir element $\mathbf{C a s}_{\mathfrak{g}}$ is insensitive to the second label $k$ of $\Lambda_{l, k}$. Atypical representations with the same value of the Casimir element are said to belong to the same block. Representations from the same block may appear within larger indecomposables, in particular they make up the projective covers $\mathcal{P}_{\Lambda_{l, k}}$. The composition series of these indecomposables are given in eqs. (A.12)-(A.15).

Let us turn our attention to the Lie superalgebra osp $(3 \mid 2)$. In this case, the bosonic subalgebra $\mathfrak{s o}(3) \oplus \mathfrak{s p}(2)$ has rank two and hence generic representations are labeled by a pair $[q, p]$ of weights. The atypicals $\lambda_{0}$ and $\lambda_{q}=[q, 2 q-1], q \geq 1 / 2$, form a 1-parameter family of representations that satisfy a single shortening condition. The label $\lambda_{0}$ is reserved for the trivial representation, $\lambda_{1 / 2}$ is the 5 -dimensional fundamental. In the case of $\mathfrak{o s p}(3 \mid 2)$, the adjoint is not atypical. Its label is $\lambda_{\mathrm{ad}}=[1,0]$.

In the representation $[q, p]$ the quadratic Casimir element $\mathbf{C a s}_{\mathfrak{h}}$ of $\mathfrak{o s p}(3 \mid 2)$ takes the values

$$
\operatorname{Cas}_{\mathfrak{h}}([q, p])=(p+2 q)(p-2 q+1) .
$$

We see that it vanishes for atypicals $\lambda_{q}$. All these atypicals belong to the unique single block from which indecomposables can be built. Once again, the most relevant indecomposables are the projective covers $\mathcal{P}_{\lambda_{q}}$ of atypicals. Their composition series are displayed in eqs. (A.12)-(A.15).

With these notations set up we can begin to construct vertex operators. Our goal is to find all vertex operators with up to two derivatives that transform in $\frac{1}{2}$ BPS representations $\Lambda_{l, k}$ of $\mathfrak{o s p}(4 \mid 2)$. Let us start with the zero modes. By definition, these fields have vanishing scaling dimension at $R=\infty$ so they cannot contain any currents $j$ or $\bar{\jmath}$. Consequently, the $\mathfrak{o s p}(3 \mid 2)$ representations $\mu, \bar{\mu}$ and $\lambda$ that label our vertex operators (2.8) are all trivial. Thus, the head must be taken from

$$
\Gamma_{0}=\Gamma_{\lambda_{0}}=\bigoplus_{l=0}^{\infty} \Lambda_{l, 0}
$$

where $\Lambda_{l, 0}=\frac{1}{2}[l+1, l-1,-l-1]$ for $l>0$ and $\Lambda_{0,0}$ is the trivial representation. In order to find the decomposition displayed on the right hand side, we employed the decomposition formulas (C.1)-(C.3) along with the fundamental results (2.11). The summation is over all those representations $\Lambda$ of $\mathfrak{o s p}(4 \mid 2)$ for which the restriction of $\mathcal{P}_{\Lambda}$ to the subalgebra $\mathfrak{o s p}(3 \mid 2)$ contains $\mathcal{P}_{\lambda_{0}}$. Our formulas in appendix $\mathrm{C}$ only list the decompositions for atypical representations $\Lambda=\Lambda_{l, k}$ but it is not difficult to see that typical (long) multiplets never contain $\mathcal{P}_{\lambda_{0}}$ in their decomposition. Hence, the formula (3.12) is exact, i.e. it accounts for all elements of $\Gamma_{0}$ not just for those that transform in $\frac{1}{2}$ BPS representations. Of course, the space $\Gamma_{0}$ is nothing but the space of functions on the supersphere $S^{3 \mid 2}$. Aside from the trivial representation $\Lambda_{0,0}$ of $\mathfrak{o s p}(4 \mid 2)$, which has vanishing Casimir, all other operators acquire a non-zero anomalous dimension,

$$
\delta_{R}^{(1)} h\left(V_{\Lambda_{l, 0}, \lambda_{0}}\right)=\frac{1}{2 R^{2}} \operatorname{Cas}_{\mathfrak{g}}\left(\Lambda_{l, 0}\right)=\frac{l^{2}}{2 R^{2}} .
$$


The next set of operators we would like to look at are the operators of weight $\left(h_{\infty}, \bar{h}_{\infty}\right)=$ $(1,0)$. Such operators contain a current $j$ and hence have $\mu=\lambda=\lambda_{\frac{1}{2}}$ while $\bar{\mu}=\lambda_{0}$ is trivial. Hence, the head of the operators must be taken from sections in the bundle

$$
\Gamma_{\lambda_{\frac{1}{2}}}=\Lambda_{0,1} \oplus \bigoplus_{l=1}^{\infty} \Lambda_{l, 0} \oplus \text { typicals }
$$

The decomposition on the right hand side is obtained from the formulas in appendix C, just as in the previous example. We see that one $\frac{1}{2}$ BPS section in the bundle of the fundamental representation $\lambda_{\frac{1}{2}}$ of $\mathfrak{o s p}(3 \mid 2)$ is the adjoint multiplet of $\mathfrak{o s p}(4 \mid 2)$. The corresponding fields are the Noether currents. According to our result (2.16) their one-loop anomalous dimension vanishes since both the Casimir of the fundamental $\lambda_{\frac{1}{2}}$ and the Casimir of the adjoint $\Lambda_{0,1}$ vanish. The remaining $\frac{1}{2} \mathrm{BPS}$ fields are derivatives of the zero modes. Their anomalous dimension is the same as for the zero modes themselves.

The $\frac{1}{2} \mathrm{BPS}$ spectrum of operators of weight $\left(h_{\infty}, \bar{h}_{\infty}\right)=(1,1)$ is a bit richer. In this case, our operators must contain $j$ and $\bar{\jmath}$ so that $\mu=\lambda_{\frac{1}{2}}=\bar{\mu}$. In the tensor product of the two fundamental representations $\mu$ and $\bar{\mu}$ we find $\lambda=\lambda_{0},[1,0],\left[\frac{1}{2}, 1\right]$. Hence, the zero mode contributions can come from 3 different bundles. The decomposition of the bundle $\Gamma_{0}$ was described in eq. (3.12) already. So it remains to describe the two bundles

$$
\Gamma_{[1,0]}=2 \Lambda_{0,1}+\Lambda_{0,2}+\text { typicals }
$$

and

$$
\Gamma_{\left[\frac{1}{2}, 1\right]}=\sum_{l=2}^{\infty}\left(2 \Lambda_{l, 0}+\Lambda_{l, 1}+\Lambda_{l,-1}\right)+\text { typicals . }
$$

If we sum up all the contributions from the three possible bundles, we find that the spectrum of operators of weight $\left(h_{\infty}, \bar{h}_{\infty}\right)=(1,1)$ decomposes into

$$
\Gamma_{\lambda_{\frac{1}{2}} \otimes \lambda_{\frac{1}{2}}} \cong \Lambda_{0,0}+2 \Lambda_{0,1}+\Lambda_{0,2}+\Lambda_{1,0}+\sum_{l=2}^{\infty}\left(3 \Lambda_{l, 0}+\Lambda_{l, 1}+\Lambda_{l,-1}\right)+\text { typicals } .
$$

The one-loop anomalous dimension of the corresponding operators is determined by the first label of the representation,

$$
\delta_{R}^{(1)} h=\frac{1}{2 R^{2}} \operatorname{Cas}_{\mathfrak{g}}\left(\Lambda_{l, k}\right)=\frac{l^{2}}{2 R^{2}} .
$$

We see in particular that our sigma model contains 145 operators with vanishing one-loop anomalous dimension. These sit in four different representations of $\mathfrak{o s p}(4 \mid 2)$. There is one state in the trivial representation $\Lambda_{0,0}$. This is the sigma model interaction that remains marginal at one-loop. It actually remains marginal at all loops. In addition, there are two adjoint multiplets $\Lambda_{0,1}$ of dimension 17 each. The multiplicity two is actually a signature of the distinction between projective covers and irreducibles. As we explained above, one could have expected that the multiplicity of the adjoint $\mathfrak{o s p}(4 \mid 2)$ section in the bundle associated to the adjoint representation $[1,0]$ of $\mathfrak{o s p}(3 \mid 2)$ is given by the number of times the 
12-dimensional $[1,0]$ appears in the decomposition of the 17 -dimensional $\Lambda_{0,1}$. Clearly, this multiplicity is one which is not the correct answer for the number of $\Lambda_{0,1}$ multiplets in $\Gamma_{[1,0]}$. So indeed the example illustrates nicely how important it is to determine the multiplicity of short operators using decompositions of projective covers rather than irreducibles.

\subsection{An alternative construction of vertex operators}

In order to fully appreciate the results of the previous subsection and the elegance of their derivation, we would like to compare our findings with more conventional constructions of vertex operators from the fundamental field multiplet $X$. In doing so, we will have to struggle a little bit with the implications of the constraint (3.1) and the equations of motion (3.4) on counting coset fields. As a reward, we will understand e.g. that the number 145 of operators with vanishing one-loop anomalous dimension contains non-trivial information about the dynamics of the supersphere sigma model.

In building coset fields from the fundamental field multiplet $X$ we shall start with the zero modes. For $h_{\infty}=\bar{h}_{\infty}=0$ the relevant fields contain no derivatives and they are given by monomials $F_{l, 0}(X)$ of order $l=0,1,2, \ldots$ in the components of $X$. Once we implement the constraint $X^{2}=1$ the components of $F_{l, 0}(X)$ transform in the traceless symmetric tensor representations $\Lambda_{l, 0}$. This agrees with our formula (3.12) above.

Let us now proceed to fields of weight $\left(h_{\infty}, \bar{h}_{\infty}\right)=(1,0)$. These must be of the form

$$
F_{l, 0}(X) \partial X
$$

for $l=0,1,2, \ldots$. The space of such objects transforms in the tensor product $\Gamma_{0} \otimes \Lambda_{1,0}$ of symmetric traceless tensors with the fundamental $\Lambda_{1,0}$. But not all these fields are non-zero. In fact, by taking a derivative of the constraint $X^{2}=1$ we obtain

$$
X \cdot \partial X=X_{a} \partial X^{a}=0 .
$$

Consequently any field of the form $F_{l, 0}(X) X \cdot \partial X$ vanishes. Such fields transform in the representation $\Gamma_{0}$. If we remove them from the list (3.19) we end up with a space of fields transforming in

$$
\Gamma_{0} \otimes \Lambda_{1,0}-\Gamma_{0}=\Lambda_{0,1}+\sum_{l=1}^{\infty} \Lambda_{l, 0}+\text { typicals }=\Gamma_{\lambda_{\frac{1}{2}}} .
$$

This agrees with our result (3.14). We have already interpreted the corresponding fields as the Noether currents and derivatives of the zero modes.

Let us now turn to the most interesting set of fields, those with weights $h=1=\bar{h}$. In this case, the counting will be affected by the equations of motion. The relevant fields can all be written in either of the following forms

$$
F_{l, 0}(X) \partial \bar{\partial} X, \quad F_{l, 0}(X) \partial X \bar{\partial} X .
$$

Our analysis of the space of these operators will proceed in two steps. First we shall fully implement the constraint $X^{2}=1$ and then we consider the equations of motion. By taking derivatives of the constraint $X^{2}=1$ we obtain the two equations

$$
X \cdot \partial X=0=X \cdot \bar{\partial} X .
$$


We can multiply each of these two equations with one of the previously found operators of dimension $\left(h_{\infty}, \bar{h}_{\infty}\right)=(1,0)$ or $\left(h_{\infty}, \bar{h}_{\infty}\right)=(0,1)$, respectively. All such operators vanish. As we discussed above, they transform in $2 \Gamma_{\lambda_{\frac{1}{2}}}$. Additionally, we also need to remove all operators created from the zero modes by multiplication with the operator $X \cdot \partial X X \cdot \bar{\partial} X$. These transform in $\Gamma_{0}$. This is not quite the end of story. In fact, there is another family of operators that vanishes because of the constraint $X^{2}=1$. To see this, we differentiate the constraint $X^{2}=1$ by $\partial \bar{\partial}$ and obtain

$$
\partial X \cdot \bar{\partial} X=-X \cdot \partial \bar{\partial} X
$$

This constraint allows us to remove all the operators of the form $F_{l, 0}(X) \partial X \cdot \bar{\partial} X$. In other words when considering the second family in eq. (3.22), we can restrict to those operators for which $\partial X \bar{\partial} X$ transforms either in the representation $\Lambda_{2,0}$ (symmetric traceless) or in $\Lambda_{0,1}$ (antisymmetric). Putting all this together we find

$$
\begin{aligned}
\Gamma_{0} \otimes \Lambda_{1,0} & +\Gamma_{0} \otimes\left(\Lambda_{2,0}+\Lambda_{0,1}\right)-2 \Gamma_{\lambda_{\frac{1}{2}}}-\Gamma_{0} \\
& =\Lambda_{0}+3 \Lambda_{0,1}+\Lambda_{0,2}+2 \Lambda_{1,0}+\sum_{l=2}^{\infty}\left(4 \Lambda_{l, 0}+\Lambda_{l, 1}+\Lambda_{l,-1}\right)+\text { typicals } \\
& =\Gamma_{\lambda_{\frac{1}{2}} \otimes \lambda_{\frac{1}{2}}}+\Lambda_{0,1}+\sum_{l=1}^{\infty} \Lambda_{l, 0}+\text { typicals }
\end{aligned}
$$

A quick glance at eq. (3.17) shows that we obtained more than we expected. The reason is simple. While we have correctly implemented the constraint $X^{2}=1$, operators of weight $\left(h_{\infty}, \bar{h}_{\infty}\right)=(1,1)$ are the first ones to be sensitive to the equations of motion. The latter precisely remove the unwanted multiplets. In the block of the zero, for example, the operators

$$
X_{I} \partial \bar{\partial} X_{J}-X_{J} \partial \bar{\partial} X_{I}
$$

contribute one of the three $\Lambda_{0,1}$ in the decomposition we have listed. Once we insert the equations of motion, however, these operators are set to zero

$$
X_{I} \partial \bar{\partial} X_{J}-X_{J} \partial \bar{\partial} X_{I}=\partial X \cdot \bar{\partial} X\left(X_{I} X_{J}-X_{J} X_{I}\right)=0 .
$$

Hence, the fact that we found 145 operators of weight $\left(h_{\infty}, \bar{h}_{\infty}\right)=(1,1)$ with vanishing one-loop anomalous dimension is sensitive to the equations of motion. Without them there would be 17 additional such operators.

\section{Duality with the osp $(4 \mid 2)$ Gross-Neveu model}

One lesson which has been learned through past studies of sigma models is that they should not be considered as an isolated research topic. There exist other important constructions of $2 \mathrm{D}$ (conformal) field theories which are intimately tied to sigma models and sometimes can provide intriguing insights into the non-perturbative features of sigma models. We have already alluded to the example of sigma models on Calabi-Yau spaces which possess a dual description in terms of (products of) WZNW coset models. Another, more 
elementary, example is the compactified free boson which admits a dual description in terms of two Majorana fermions. The proposed duality between the sigma model on $S^{3 \mid 2}$ and the $\mathfrak{o s p}(4 \mid 2)$ Gross-Neveu model that we described in the introduction is quite similar to the Coleman-Mandelstam duality between bosons and fermions only that the abelian symmetry $\mathfrak{u}(1)=\mathfrak{s o}(2)$ is replaced by the non-abelian $\mathfrak{o s p}(4 \mid 2)$.

In the first subsection we shall describe the $\mathfrak{o s p}(4 \mid 2)$ Gross-Neveu model and some of its most basic features. Then we review a central all-loop result from [13] on the (target space) $\frac{1}{2}$ BPS spectrum of perturbed supergroup WZNW models and explain how it applies to the $\mathfrak{o s p}(4 \mid 2)$ Gross-Neveu model. In the third subsection we try to match the $\frac{1}{2} \mathrm{BPS}$ spectrum of the Gross-Neveu model for a certain value of the Gross-Neveu coupling to the one-loop spectrum of the supersphere sigma models. We will find perfect agreement for low lying states, but also some discrepancies that involve fields with more derivatives. The discussion of these findings is mostly deferred to the final section.

\subsection{The $\mathfrak{o s p}(4 \mid 2)$ Gross-Neveu model}

The fundamental field multiplet $\Psi=\left(\Psi_{A}\right)=\left(\psi_{j}, \gamma_{a}\right)$ of the $\mathfrak{o s p}(4 \mid 2)$ Gross-Neveu model consists of four Majorana fermions $\psi_{j}, j=1, \ldots, 4$, and a bosonic $\beta \gamma$-system whose fields we shall denote by $\gamma_{1}=\gamma$ and $\gamma_{2}=\beta$. In addition, there is a second multiplet $\bar{\Psi}=\left(\bar{\psi}_{j}, \bar{\gamma}_{a}\right)$ of opposite chirality. All these six fields in $\Psi$ possess conformal weight $h=1 / 2$ and transform in the fundamental representations $\Lambda_{1,0}$ of $\mathfrak{o s p}(4 \mid 2)$. The same applies to $\bar{\Psi}$. In terms of these field multiplets, the action of the Gross-Neveu model reads

$$
\begin{aligned}
& \mathcal{S}^{\mathrm{GN}}[\psi, \gamma, \bar{\psi}, \bar{\gamma}]=\frac{1}{2 \pi} \int d^{2} z {\left[\sum_{j}\left(\psi_{j} \bar{\partial} \psi_{j}+\bar{\psi}_{j} \partial \bar{\psi}_{j}\right)+\left(\gamma_{2} \bar{\partial} \gamma_{1}+\bar{\gamma}_{2} \partial \bar{\gamma}_{1}\right)\right] } \\
&+\frac{g}{2 \pi} \int d^{2} z\left[\sum_{j} \psi_{j} \bar{\psi}_{j}+\left(\gamma_{1} \bar{\gamma}_{2}-\gamma_{2} \bar{\gamma}_{1}\right)\right]^{2}
\end{aligned}
$$

The $\mathfrak{o s p}(4 \mid 2)$ invariance of this action is manifest since all indices are contracted with the $\mathfrak{o s p}(4 \mid 2)$ invariant metric. When written in terms of $\Psi$ and $\bar{\Psi}$, rather than its components, the action takes the same form as that of the massless Thirring model with its characteristic fourth order interaction term. When the coupling constant $g$ is set to zero the model is free and scale invariant. It possesses a Virasoro symmetry with central charge $c=1$. The latter receives a contribution $c_{j}=1 / 2$ from each of the fermions $\psi_{j}$ and $c_{a}=-1 / 2$ from the two components of the $\beta \gamma$-system. Switching on the coupling $g$ introduces a very non-trivial action but it turns out to preserve conformal symmetry. In fact, the $\beta$-function for the coupling $g$ is proportional to the dual Coxeter number $h^{\vee}=\operatorname{Cas}_{\mathfrak{g}}\left(\Lambda_{0,1}\right)$ and hence vanishes for $\mathfrak{o s p}(4 \mid 2)$. Therefore, the $\mathfrak{o s p}(4 \mid 2)$ Gross-Neveu model defines a one-parameter family of interacting conformal field theories with central charge $c=1$.

While the interaction in the $\mathfrak{o s p}(4 \mid 2)$ Gross-Neveu model preserves the Virasoro and a global $\mathfrak{o s p}(4 \mid 2)$ symmetry, the free field theory possesses additional current algebra symmetries that are broken when $g \neq 0$. In order to describe these symmetries, we recall that the components of the field multiplet $\Psi$ obey the following operator product expansions

$$
\psi_{i}(z) \psi_{j}(w) \sim \frac{\delta_{i j}}{z-w}+\ldots, \quad \gamma_{2}(z) \gamma_{1}(w) \sim \frac{\delta_{a b}}{z-w} .
$$


Using these operator product expansions between the fundamental constituents it is standard to show that the following quadratic combinations

$$
J_{A B}=\Psi_{A} \Psi_{B} \quad \text { where } \quad\left(\Psi_{A}\right)=\left(\psi_{i}, \gamma_{b}\right)
$$

obey the algebraic relations of an $\mathfrak{o s p}(4 \mid 2)$ current algebra at level $k=1$. Let us stress once again that this current algebra symmetry is broken as soon as we switch on the coupling.

The current algebra symmetry suggests interpreting the free theory at $g=0$ as a WessZumino-Novikov-Witten (WZNW) model. In addition, it is not difficult to verify that the fourth order interaction term of the Gross-Neveu model can be expressed in terms of the currents (4.3) as

$$
\frac{g}{2 \pi} \int d^{2} z\left[\sum_{i} \psi_{i} \bar{\psi}_{i}+\gamma_{1} \gamma_{2}-\gamma_{2} \bar{\gamma}_{1}\right]^{2}=\frac{g}{2 \pi} \int d^{2} z \sum_{A B} J_{A B}(z) \bar{J}^{A B}(\bar{z})
$$

Putting all this together we have shown that the Gross-Neveu model can be thought of as a deformed WZNW model at level $k=1$,

$$
\mathcal{S}^{\mathrm{GN}}=\mathcal{S}_{k=1}^{\mathrm{WZNW}}+\frac{g}{2 \pi} \int d^{2} z \sum_{A B} J_{A B}(z) \bar{J}^{A B}(\bar{z})
$$

with the deformation being generated by an exactly marginal current-current interaction. This reformulation of the $\mathfrak{o s p}(4 \mid 2)$ Gross-Neveu model will become important when we apply the powerful results of [13] to the Gross-Neveu model.

\subsection{An all-loop result for deformed WZNW models}

In [13], current-current deformations of supergroup WZNW models were studied. In particular it was argued that the deformation by the operator

$$
\Omega(z, \bar{z})=J^{\mu}(z) \bar{J}_{\mu}(\bar{z}),
$$

is truly marginal, provided that the Lie supergroup possesses vanishing dual Coxeter number, i.e. that $G=\operatorname{PSL}(N \mid N), \operatorname{OSP}(2 N+2 \mid 2 N), \mathrm{D}(2,1 ; \alpha)$. In the definition of $\Omega$ the sum runs over all directions $\mu$ in the Lie superalgebra $\mathfrak{g}$. The deformation breaks the affine symmetry. Since it does not even commute with the zero modes of the chiral currents, it also breaks the left and right $\mathfrak{g}$ symmetries. On the other hand, the sum of left and right zero modes does commute with the perturbing operator so that the deformed theory preserves the diagonal $\mathfrak{g}$ action.

Of course, under the perturbation with the operator (4.6) the conformal weight of fields can change, i.e. fields may develop an anomalous dimension. In general, this anomalous dimension is difficult to compute, at least beyond the leading order in perturbation theory. Remarkably, for a special subset of fields, the authors of [13] managed to obtain an all order expression. In physics terminology, the fields for which this was possible are those that transform in maximally atypical, or $\frac{1}{2} \mathrm{BPS}$, representations of the target space symmetry $\mathfrak{g}$. More precisely, the formulas of [13] hold for all indecomposable field multiplets of $\mathfrak{g}$ which 
contain a subrepresentation of non-zero superdimension. For such fields, the anomalous dimension reads

$$
\delta_{g}^{(\infty)} h_{\mathrm{BPS}}=\frac{g}{2\left(1-k^{2} g^{2}\right)}\left[\operatorname{Cas}_{\mathfrak{g}}^{D}\left(\Lambda_{\mathrm{BPS}}\right)-(1-k g)\left(\mathbf{C a s}_{\mathfrak{g}}^{L}+\mathbf{C a s}_{\mathfrak{g}}^{R}\right)\right] .
$$

Here $\mathbf{C a s}_{\mathfrak{g}}^{L / R}$ refers to the value of the quadratic Casimirs on the left and right representations in the unperturbed model, respectively. The superscript $D$ means that the Casimir element is evaluated with respect to the diagonal action. We have placed the subscript 'BPS' on both sides of the equation to remind us that this formula should only be applied to fields that transform in maximally atypical representations $\Lambda$ under the diagonal action. On the other hand, their transformation law with respect to left or right action in the WZNW model is not constrained.

Let us now specialize this very general result to the $\mathfrak{o s p}(4 \mid 2)$ Gross-Neveu model or, equivalently, to the current-current deformation of the $\mathfrak{o s p}(4 \mid 2)$ WZNW model at level $k=1$. In this case our formula can be applied to all fields that transform in one of the atypical representations $\Lambda_{l, k}$ or any indecomposable composites formed from these. Let us recall that the value of the quadratic Casimir element assumes the value $\mathbf{C a s}_{\mathfrak{g}}\left(\Lambda_{l, k}\right)=l^{2}$ on such atypicals. Hence, our general formula (4.7) becomes

$$
\delta_{g}^{(\infty)} h_{\mathrm{BPS}}=\frac{g l^{2}}{2\left(1-g^{2}\right)}-\frac{g}{2(1+g)}\left(\mathbf{C a s}_{\mathfrak{g}}^{L}+\mathbf{C a s}_{\mathfrak{g}}^{R}\right)
$$

for fields transforming in $\Lambda=\Lambda_{l, k}$ with respect to the diagonal action of $\mathfrak{g}$. Note that the function $\delta_{g}^{(\infty)} h$ develops a singularity at $g=-1$, at least for a large number of states. This simple observation motivates the identification of the point $g=-1$ with the $R \rightarrow \infty$ limit of the $S^{3 / 2}$ sigma model. In fact, in the sigma model one expects that all winding states develop infinite energy when $R \rightarrow \infty$. So, if we want the sigma model to be dual to the Gross-Neveu model, we are forced to identify $g=-1$ with the infinite radius limit. The precise relation between the coupling $g$ and the radius $R$ reads $[13]^{1}$

$$
g=\frac{4-R^{2}}{4+R^{2}}
$$

For a state to remain in the spectrum at the point $g=-1$, the anomalous dimension (4.8) has to remain finite. This is the case if

$$
\operatorname{Cas}_{\mathfrak{g}}^{L}+\operatorname{Cas}_{\mathfrak{g}}^{R}=\frac{l^{2}}{2} .
$$

We call eq. (4.10) the no-winding condition. For states that satisfy this condition, the anomalous dimension (4.8) simplifies to

$$
\delta_{g}^{(\infty)} h_{\mathrm{BPS}}=\frac{1}{4} \frac{g l^{2}}{1-g}=-\frac{l^{2}}{8}+\frac{l^{2}}{2 R^{2}} .
$$

\footnotetext{
${ }^{1}$ The cohomological methods developed in [39] imply that the relation is identical to the one that appears in the duality between a compactified free boson and the massless Thirring model.
} 
Here we also inserted eq. (4.9) so that the anomalous dimension of the Gross-Neveu model fields is finally written in terms of the radius parameter $R$ of the sigma model. We have now gathered all the ingredients we need in order to perform our first tests of the duality. Eq. (4.10) tells us which states of the free field theory make it into the spectrum at $g=-1$ and eq. (4.11) allows us to compute the corresponding conformal weight. We will now start to compare the resulting spectrum at $g=-1$ with the free supersphere sigma model.

In our discussion of the one-loop anomalous dimensions for coset sigma models we briefly commented on a puzzling instability that arises from high gradient operators. The same type of instabilities also appears in perturbed WZNW models, at least for generic choices of the target group and the level. To leading order in perturbation theory this was observed by Ryu et al. in [34]. With the help of formula (4.7) one may show that these instabilities persist to any order in perturbation theory. The authors of [34] also observed that no instabilities occur for $\mathfrak{p s u}(N \mid N)$ WZNW models at level $k=1$. This observation, however, does not carry over to our $\mathfrak{o s p}(4 \mid 2)$ WZNW model at level $k=1$. In fact, one can show that this theory contains instabilities arbitrarily close to the free field theory, much as it is the case for sigma models. For now, we shall close an eye on these issues.

\subsection{Checking the proposed duality}

We want to apply the results on the deformation of the $\frac{1}{2}$ BPS spectrum in deformed supergroup WZNW models in order to test the proposed duality between the $\mathfrak{o s p}(4 \mid 2)$ Gross-Neveu model and the supersphere sigma model. In the first subsection we shall show that the zero mode spectrum of the sigma model is recovered along with its 1-loop deformation. This is a remarkable example of an emergent geometry. In the WZNW model, the fields that are associated with spherical harmonics of the supersphere possess very large scaling dimensions. These come down until they become zero modes, i.e. fields with vanishing scaling weight, in the sigma model limit. Let us anticipate that the singular vectors of the $\mathfrak{o s p}(4 \mid 2)$ WZNW model at level $k=1$ play an important role for this identification with the zero mode spectrum of the sigma model to work out. Then we turn to derivative fields of the sigma model. We will argue that the agreement continues to hold for fields of conformal weight $\left(h_{\infty}, \bar{h}_{\infty}\right)=(1,0),(0,1)$ in the sigma model. This may not come as a big surprise. Things become more interesting for the fields with conformal weight $\left(h_{\infty}, \bar{h}_{\infty}\right)=(1,1)$ since these are sensitive to the equations of motion in the sigma model. Recall that in the sigma model we found 145 states with vanishing 1-loop scaling dimension. This will be exactly matched by the deformed WZNW model. In the WZNW model, the scaling dimension of the corresponding 145 states is independent of the coupling so that the conjectured duality makes an interesting prediction: all higher loop corrections to the scaling weight of the 145 states are actually zero. The match between the deformed WZNW model and the sigma model extends to many other fields with $\left(h_{\infty}, \bar{h}_{\infty}\right)=(1,1)$. On the other hand, we will also find sigma model fields that cannot be reproduced within the deformed WZNW model. 


\subsubsection{Ground state spectrum}

One key piece of evidence in support of the proposed duality is the observation that we can actually recover all the zero modes of the sigma model. Under the action of the global $\mathfrak{o s p}(4 \mid 2)$ symmetry the space $\Gamma_{0}$ of functions on the supersphere decomposes into a sum of irreducible multiplets $\Lambda_{l, 0}$, see eq. (3.12). Each of these multiplets appears with multiplicity one. Other atypical representations $\Lambda_{l, k}, k \neq 0$ do not occur.

As we have explained before, the states of the Gross-Neveu model are constructed from a chiral multiplet $\Psi=\Psi^{L}$ that transforms in a 6 -dimensional representation of $\mathfrak{o s p}(4 \mid 2)$. The $\mathfrak{o s p}(4 \mid 2)$ representation matrices are those known from the usual fundamental representation, but the grading rules are reversed so that the fermionic subspace is 4-dimensional while the bosonic has dimension 2. It is a remarkable fact that the conformal dimension $h$ of all chiral operators $\mathcal{O}^{L}$ in the undeformed case is bounded from below by

$$
h_{0}\left(\mathcal{O}_{[\Lambda]}^{L}\right) \geq \frac{1}{2} \operatorname{Cas}_{\mathfrak{g}}^{L}(\Lambda),
$$

for all $\mathcal{O}^{L}$ that transform in the representation $[\Lambda]$ with respect to the left $\mathfrak{o s p}(4 \mid 2)$ action. Of course, the corresponding statement holds for all operators $\mathcal{O}^{R}$ that are constructed from the components of $\bar{\Psi}=\Psi^{R}$ and their derivatives. It is actually possible to establish the stronger lower bound

$$
h_{0}\left(\mathcal{O}_{[\Lambda]}^{L}\right) \geq j_{1}+j_{2}\left(j_{2}+1\right)+j_{3}\left(j_{3}+1\right)+\left|j_{2}-j_{3}\right| \geq \frac{1}{2} \operatorname{Cas}_{\mathfrak{g}}^{L}(\Lambda)
$$

which shows that the inequality (4.12) can only be saturated by very special multiplets, when $j_{1}=0, \frac{1}{2}$. It turns out that for each integer $l=0,1,2, \ldots$ there is a unique field multiplet $\mathcal{O}_{l}^{L}$ such that

$$
h_{0}\left(\mathcal{O}_{l}^{L}\right)=\frac{l^{2}}{2} .
$$

The multiplet $\mathcal{O}_{l}^{L}$ is obtained as a graded symmetric component in the $l$-fold tensor product of the fundamental. Since our generating field multiplet $\Psi$ is fermionic, i.e. its grading is reversed in comparison to the grading of the fundamental, the multiplet $\mathcal{O}_{l}^{L}$ must contain $l(l-1) / 2$ derivatives. Hence, its conformal dimension $h\left(\mathcal{O}_{l}^{L}\right)=l / 2+l(l-1) / 2=l^{2} / 2$.

Let us illustrate the construction of $\mathcal{O}_{l}^{L}$ with a few explicit examples. Of course, the operator $\mathcal{O}_{0}^{L}$ is just the identity field while $\mathcal{O}_{1}^{L}$ is the fundamental multiplet $\Psi$. The next multiplet $\mathcal{O}_{2}^{L}$ appears at $h\left(\mathcal{O}_{2}^{L}\right)=2$,

$$
\mathcal{O}_{2}^{L}=\left(\psi_{A} \partial \psi_{B}+(-1)^{|A||B|} \psi_{B} \partial \psi_{A}\right)
$$

When we multiply the multiplet $\mathcal{O}_{l}^{L}$ with its anti-holomorphic partner $\mathcal{O}_{l}^{R}$ we obtain a set of bulk fields which transform in the product $\Lambda_{l, 0} \otimes \Lambda_{l, 0}$. The only component that can satisfy the no-winding condition is the one in the representation $\Lambda_{2 l, 0}$. Indeed,

$$
\operatorname{Cas}_{\mathfrak{g}}\left(\Lambda_{2 l, 0}\right)=4 l^{2}=2\left(\operatorname{Cas}_{\mathfrak{g}}^{L}\left(\Lambda_{l, 0}\right)+\operatorname{Cas}_{\mathfrak{g}}^{R}\left(\Lambda_{l, 0}\right)\right) .
$$

Let us denote the this component of the product by $V_{2 l}=V_{2 l}(z, \bar{z})$. To summarize, we have now constructed a field multiplet $V_{2 l}$ in the WZNW model that transforms in the 
representation $\Lambda_{l, 0}$ with respect to both the left and the right action of $\mathfrak{o s p}(4 \mid 2)$ and in the representations $\Lambda_{2 l, 0}$ with respect to the diagonal action. In the WZNW model, i.e. the free Gross-Neveu model, this field possesses weights $\left(h_{0}\left(V_{2 l}\right), \bar{h}_{0}\left(V_{2 l}\right)\right)=\left(l^{2} / 2, l^{2} / 2\right)$.

Since the field $V_{2 l}$ transforms on the $\frac{1}{2} \mathrm{BPS}$ representation $\Lambda_{2 l, 0}$ of $\mathfrak{o s p}(4 \mid 2)$, we can apply the results of the previous subsection to compute its dimension for any value of the coupling $g$ and in particular at the point $g=-1$. With the help of the leading term in eq. (4.11) we obtain

$$
h\left(V_{2 l}\right)_{g=-1}=h_{0}\left(V_{2 l}\right)-\frac{1}{8} 4 l^{2}=0 .
$$

Hence, we obtain precisely the spectrum provided by the spherical harmonics $\Lambda_{2 l, 0}$ in the sigma model, i.e. at least one half of the zero modes of the supersphere sigma model. ${ }^{2}$ Remarkably, this identification is also consistent with what we know about the 1-loop anomalous dimensions in the sigma model. In fact, if we keep the next to leading term in eq. (4.11) we find

$$
h\left(V_{2 l}\right)_{g}=\frac{l^{2}}{2}+\frac{g l^{2}}{1-g}=\frac{2 l^{2}}{R^{2}} .
$$

This should be compared with the result (3.13) for the one loop anomalous dimension of the sigma model vertex operators $V_{\Lambda_{2 l, 0}, \lambda_{0}}$. We see that also the 1-loop corrections to the scaling law agree. In the deformed WZNW model, the formula (4.11) is actually exact, i.e. there are no further corrections by terms involving higher powers of the sigma model coupling $1 / R^{2}$. The duality therefore predicts that the anomalous dimensions of zero mode fields in the sigma model are 1-loop exact. It should not be too difficult to check this prediction through a direct computation along the lines of [40,41], where anomalous dimensions of tachyonic vertex operators in bosonic $\mathrm{O}(\mathrm{N})$ models were computed up to four loops. The general structure of Wegner's results suggest that higher order corrections indeed vanish for the conformal supersphere models, but we have not yet completed an honest derivation.

Since our fields $\mathcal{O}_{l}^{L / R}$ are the only ones satisfying the bound (4.12) and the bulk field $V_{2 l}$ the only fields we could build from them that solve the no-winding condition (4.10), the deformed WZNW model contains no further field of weight $\left(h_{\infty}, \bar{h}_{\infty}\right)=(0,0)$ at $g=-1$. Moreover, because of the bound (4.12), all other WZNW fields that solve the no-winding condition end up with $h_{\infty}+\bar{h}_{\infty}>0$ for $g=-1$. In the free sigma model, the conformal weights are determined by the number of derivatives and hence they are certainly nonnegative. So, our results are in beautiful agreement with the proposed duality.

Let stress that the match of zero modes only works for the WZNW model at $k=1$, i.e. it does make crucial use of the exact position of singular vectors. In order to illustrate this point let us consider the space of states $\mathcal{H}_{k}^{(l)}$ of conformal weight $h=2(\bar{h}=0)$. For an $\mathfrak{o s p}(4 \mid 2)$ WZNW model with $k>1$, these transform in

$$
\mathcal{H}^{(2)} \cong \Lambda_{0,1}+\Lambda_{0,1} \odot \Lambda_{0,1}=\Lambda_{0,0}+\Lambda_{0,1}+\Lambda_{2,-1}+2 \Lambda_{2,0}+\Lambda_{2,1}+[2,0,0] .
$$

\footnotetext{
${ }^{2}$ One would expect to obtain the missing zero modes $V_{2 l+1}$ from other sectors of the Gross-Neveu model. Without the inclusion of additional states, the Gross-Neveu model is related to an orbifold theory $S^{3 / 2} / \mathbb{Z}_{2}$ rather than the supersphere sigma model.
} 
The term $\Lambda_{0,1}$ originates from the action of the modes $J_{-2}^{A B}$ while the term $\Lambda_{0,1} \odot \Lambda_{0,1}$ contains the contributions of $J_{-1}^{A B} J_{-1}^{C D}|0\rangle$. A formula for the symmetric tensor product $\odot$ of the adjoint $\Lambda_{0,1}$ can be found at the end of appendix $A$. Note that there appear four different multiplets in which the Casimir element has the maximal value $\operatorname{Cas}_{\mathfrak{g}}(\Lambda)=4$, namely the multiplets $\Lambda=\Lambda_{2, k}, k=0, \pm 1$. At level $k=1$, the first singular vectors appear at $h=2$ and these reduce the spectrum to

$$
\mathcal{H}_{k=1}^{(2)} \cong \Lambda_{0,0}+\Lambda_{0,1}+\Lambda_{2,0}+[2,0,0]
$$

so that the representations with maximal Casimir are reduced to a single one, namely $\Lambda_{2,0}$. This is the unique multiplet in $\mathcal{H}_{k=1}^{(2)}$ that is used to build a zero mode at $g=-1$. WZNW models with level $k>1$ contain many more zero modes and hence cannot be dual to the supersphere sigma model.

\subsubsection{Spectrum of gradient operators}

After our success in matching the zero modes of the sigma model with fields in the deformed WZNW theory, we want to move on to gradient fields in the sigma model. Some of them are very easy to find. This applies in particular to the operators of weight $\left(h_{\infty}, \bar{h}_{\infty}\right)=(1,0)$. Their spectrum was described in eq. (3.14). Most of these fields emerge from the WZNW model derivative operators $\partial V_{2 l}$ with $l=1,2, \ldots$ The fields $V_{2 l}$ were constructed in the previous subsection. The bulk operators $\partial V_{2 l}$ have conformal weight $\left(h_{0}, \bar{h}_{0}\right)=\left(l^{2} / 2+1, l^{2} / 2\right)$ and they transform in the representation $\Lambda_{2 l, 0}$. By the same reasoning as above we obtain a family of fields with weight $\left(h_{\infty}, \bar{h}_{\infty}\right)=(1,0)$ at the point $g=-1$ which transform in the $\Lambda_{2 l, 0}$ representations of $\mathfrak{o s p}(4 \mid 2)$. Their 1-loop anomalous dimension coincides with that of the corresponding zero modes. Of course, the match with the operators of weight $\left(h_{\infty}, \bar{h}_{\infty}\right)=(1,0)$ is not surprising since they are obtained as derivatives in both the WZNW and the sigma model description.

There is one more set of operators at $\left(h_{\infty}, \bar{h}_{\infty}\right)=(1,0)$, namely the Noether currents of the sigma model that sit in the representation $\Lambda_{0,1}$. It is obvious that these arise from the chiral currents $J^{A B}$ in the WZNW model. In fact, the currents of the WZNW model transform in the representation $\Lambda^{L}=\Lambda_{0,1}$ and $\Lambda^{R}=\Lambda_{0,0}$ with respect to the left and right action of $\mathfrak{o s p}(4 \mid 2)$, respectively. Under the diagonal action, the transformation law is described by the tensor product $\Lambda^{D}=\Lambda_{0,1} \otimes \Lambda_{0,0}=\Lambda_{0,1}$. Since all these representations possess vanishing Casimir, the no-winding condition (4.10) is satisfied and the anomalous contribution to the conformal weight vanishes. Hence, we can identify the deformation of the WZNW currents with the Noether currents of the sigma model.

Let us now turn to the operators of conformal weight $\left(h_{\infty}, \bar{h}_{\infty}\right)=(1,1)$ in the sigma model. Their spectrum in the sigma model is given by eq. (3.17). Obviously, we can obtain some of these from the operators $\partial \bar{\partial} V_{2 l}, l=1,2, \ldots$ in the WZNW model. But these fields are not even close to exhausting the content of eq. (3.17). In particular, the sigma model contains these 145 marginal fields with vanishing 1-loop anomalous dimension that we discussed extensively in section 3 and so far we have not seen any of them.

These 145 fields belong to mutiplets $\Lambda_{0,0}+2 \Lambda_{0,1}+\Lambda_{0,2}$, all of which have vanishing Casimir. Hence, in the WZNW model they must appear with $\left(h_{0}, \bar{h}_{0}\right)=(1,1)$. So, let 
us count the fields in the WZNW model that have weights $\left(h_{0}, \bar{h}_{0}\right)=(1,1)$ and vanishing Casimir. All of these fields must arise among $J_{A} \bar{J}_{B}$, i.e. sit in the tensor product of the adjoint representation of $\mathfrak{o s p}(4 \mid 2)$ with itself. This tensor product is given by

$$
\Lambda_{0,1} \otimes \Lambda_{0,1} \cong \Lambda_{0,0}+2 \Lambda_{0,1}+\Lambda_{0,2}+\Lambda_{2,-1}+2 \Lambda_{2,0}+\Lambda_{2,1}+[2,0,0] .
$$

Indeed, this contains exactly 145 fields in representations from the block of the trivial representations for which the anomalous dimension vanishes to all orders in the coupling and hence also around $g=-1$, in perfect agreement with the sigma model results. Since the space of marginal fields in the sigma model is truncated by the equations of motion, the deformed WZNW model has the sigma model equations of motion built in!

This is a remarkable agreement. On the other hand, looking back at the sigma model spectrum (3.17) we realize that the content of what looks like $\mathcal{P}_{\Lambda_{2 l, 0}}, l=1,2, \ldots$ is still missing. Additional fields in these representations that acquire weights $\left(h_{\infty}, \bar{h}_{\infty}\right)=(1,1)$ at $g=-1$ do exist in the WZNW, but these turn out not to match the 1-loop data near $g=-1$. This is the first discrepancy between the Gross-Neveu and the sigma model. We shall discuss this and other discrepancies in more detail in the concluding section.

Before we do so, let us point out that, once again, the singular vectors are absolutely crucial in order for the WZNW model to respect the sigma model equations of motion. As an example let us look at the operators of the form $\partial \bar{\partial} V_{4}$. These give rise to a single marginal sigma model field in the representation $\Lambda_{4,0}$. If it was not for the singular vectors of conformal weight $h=2$, the WZNW model would give many more marginal fields in the same block. In fact, the tensor product

$$
\left(2 \Lambda_{2,0}+\Lambda_{2,1}+\Lambda_{2,-1}\right) \otimes\left(2 \Lambda_{2,0}+\Lambda_{2,1}+\Lambda_{2,-1}\right) \cong \Lambda_{4,-2}+4 \Lambda_{4,-1}+6 \Lambda_{4,0}+4 \Lambda_{4,1}+\Lambda_{4,2}+\ldots
$$

where $+\ldots$ stand for multiplets $\Lambda$ with $\operatorname{Cas}_{\mathfrak{g}}(\Lambda)<16$, none of which satisfy the nowinding condition. But those that do clearly outnumber the spectrum of marginal sigma model fields.

\section{Conclusions}

In this work we have reviewed recent results on the spectrum of coset sigma models and applied them to the conformal supersphere sigma model with target space $S^{3 \mid 2}$. The example shows very clearly that the construction of vertex operators designed in [9] provides easy access to the spectrum of sigma models, at least to leading order in the sigma model coupling. We have then used the results to test a conjectured dual description of the sigma model on $S^{3 \mid 2}$ which becomes weakly coupled deep in the strongly curved regime of the sigma model. The dual theory may be regarded as an $\mathfrak{o s p}(4 \mid 2)$ Gross-Neveu model or, equivalently, a deformed $\mathfrak{o s p}(4 \mid 2)$ WZNW model at level $k=1$. With the help of all-loop results from [13] we were able to recover the zero mode spectrum of the sigma model along with a number of gradient fields. In particular, we argued that the sigma model equations of motion are implemented in the deformed WZNW model.

There are quite a few open problems associated with both the perturbative results we reviewed and with the duality. We have already explained the issue of perturbative 
instabilities from high gradient operators in sigma models, see the final remarks in section 2. These remain puzzling and there is a wide range of proposals on how they could be interpreted, including e.g. the suggestion that they might be cured by non-perturbative effects [35], or that they indicate the existence of higher fixed points [14]. High gradient instabilities are not limited to sigma models. In fact, they have also been observed to occur in perturbed WZNW models [34]. The authors of that work also noticed that high gradient instabilities are avoided for deformed $\mathfrak{p s u}(N \mid N)$ WZNW models at level $k=1$ since in this case singular vectors remove the unstable operators. This is not true for $\mathfrak{o s p}(2 N+2 \mid 2 N)$, however, which is plagued by high gradient instabilities, even at level $k=1$. Since the phenomenon appears to be so omnipresent, it seems mandatory to uncover its (ir)relevance.

The duality between the Gross-Neveu and the sigma model we studied in section 4 also leaves us with a number of interesting open questions. To begin with, let us observe that for all $\frac{1}{2} \mathrm{BPS}$ states in the sigma model that are dual to no-winding states of the WZNW model, the 1-loop anomalous dimension must be exact, i.e. it should not receive any higher loop corrections. We have actually stressed before that our formula (4.11) is exact, i.e. in its derivation we did not drop any terms of higher order in $1 / R^{2}$. The only $R$ dependent correction term agrees exactly with the 1-loop result in eq. (2.16), assuming that $\operatorname{Cas}_{\mathfrak{h}}(\mu)+\mathbf{C a s}_{\mathfrak{h}}(\bar{\mu})=0$ and inserting $\mathbf{C a s}_{\mathfrak{g}}\left(\Lambda_{l, k}\right)=l^{2}$. It would be very interesting to verify this consequence of the duality through a 2-loop computation. Some 2-loop computations for high gradient operators in sigma models were performed previously in [35]. Of course, designing an argument that establishes 1-loop exactness for the relevant subsector in the sigma model would be even more remarkable.

In the last section we have also found some sigma model fields that do not seem to possess a counterpart in the deformed WZNW model, namely a large number of fields at weight $\left(h_{\infty}, \bar{h}_{\infty}\right)=(1,1)$. These are not the only sigma model fields that cannot be matched. In fact, the comparison of eqs. (2.16) and (4.11) shows that fields for which the sum $\operatorname{Cas}_{\mathfrak{h}}(\mu)+\operatorname{Cas}_{\mathfrak{h}}(\bar{\mu}) \neq 0$ cannot possess a counterpart in the Gross-Neveu model, at least not in the sense we outlined. On the other hand, there exist intriguing further coincidences between the spectra of the two theories which we were not able to incorporate into the above analysis. In particular, the authors of [12] uncovered some miraculous character identities that establish a correspondence between all chiral fields in the sigma model, no matter how large $h_{\infty}$ or $\bar{h}_{\infty}$, and fields in the deformed WZNW model. Unfortunately, the one-loop data in the sigma model spoil this match. Of course, it is possible that these discrepancies simply disprove the duality. On the other hand, it seems somewhat tempting to speculate that the discrepancies might have the same origin as the high gradient instabilities described above. Very much in the spirit of [34] one might hope that the duality could even offer new insights into the instabilities, but so far we have not been able to make this more concrete.

On a more technological level, our work demonstrates that existing results on the spectrum of superspace sigma and WZNW models can provide very powerful tools to test dualities and to develop an efficient description of sigma models deep in the strongly coupled regime. There are many other models to which these ideas might apply. In particular, a similar duality between conformal sigma models on complex projective superspace and 
$\mathfrak{p s u}(N \mid N)$ WZNW models has been proposed at various places in the literature. ${ }^{3}$ It should also be possible to extend the perturbative computations in superspace sigma models to those target spaces that appear in the context of the AdS/CFT correspondence. This requires two generalizations of the present setup. Whereas the 1-loop results we have reviewed above are restricted to symmetric spaces $G / H$ in which $H \subset G$ is fixed by an automorphism of order two, the description of strings in AdS backgrounds involves subgroups $H \subset G$ which are held fixed by an automorphism of order four. The extension to such generalized symmetric spaces is a bit cumbersome but should not meet any fundamental difficulty. Another fundamental aspect of AdS backgrounds is that they are non-compact. This has implications on the way we construct normalizable sections, at least when the denominator group $H$ is non-compact as well. For $\mathrm{AdS}_{2}$ backgrounds, on the other hand, the construction of vertex operators reviewed above remains unaltered. We will address such compactifications in future research.

\section{Acknowledgments}

The authors wish to thank Constantin Candu, Vladimir Mitev, Andreas Ludwig, Christopher Mudry, Thomas Quella and Hubert Saleur for comments and interesting discussions. The research leading to these results has received funding from the People Programme (Marie Curie Actions) of the European Union's Seventh Framework Programme FP7/2007-2013/ under REA Grant Agreement No 317089 (GATIS).

\section{A Representation theory of $\mathfrak{o s p}(4 \mid 2)$}

In the following we give a very basic introduction to the Lie superalgebra $\mathfrak{o s p}(4 \mid 2)$ and (some of) its finite dimensional representations. The complex superalgebra $\mathfrak{g}:=\mathfrak{o s p}(4 \mid 2)$ may be realized as the set of supermatrices,

$$
\mathfrak{o s p}(4 \mid 2)=\left\{\left(\begin{array}{cc}
A & B \\
J_{2} B^{t} & D
\end{array}\right): A^{t}=-A \text { and } D^{t} J_{2}=-J_{2} D\right\} .
$$

Here $A$ is a $4 \times 4$ matrix, $D$ is a $2 \times 2$ matrix and $B$ is rectangular of size $4 \times 2$. In addition, we introduced the $2 \times 2$ matrix $J_{2}=\left(\begin{array}{cc}0 & -1 \\ 1 & 0\end{array}\right)$. As usual, the Lie superalgebra $\mathfrak{g}$ decomposes into an even, or bosonic, subalgebra $\mathfrak{g}_{0}=\mathfrak{s o}(4) \oplus \mathfrak{s p}(2) \cong \mathfrak{s l}(2) \oplus \mathfrak{s l}(2) \oplus \mathfrak{s l}(2)$ and an odd, or fermionic, subspace $\mathfrak{g}_{\overline{1}}$.

Our review of representations focuses on finite dimensional representations. As usual for superalgebras, irreducible representations fall into two different categories. On the one hand, there are the generic long multiplets. These are also known as typical representations in the more mathematical literature. On the other hand, a superalgebra also possesses short or BPS multiplets which mathematicians refer to as atypical representations. BPS multiplets can be put together into indecomposable representations. We will only work with one class of such indecomposables, namely the projective covers of atypical representations.

\footnotetext{
${ }^{3}$ The statement has been communicated in the past by H. Saleur and by A. Ludwig. One argument has been written down in [34]. A different reasoning can be found in [42]
} 
In order to make all this more precise, we note that an integral dominant highest weight $\Lambda=\left(j_{1}, j_{2}, j_{3}\right)$ of $\mathfrak{g}_{\overline{0}}$ is also one for the full superalgebra $\mathfrak{g}$ if it obeys the consistency conditions

$$
j_{1}=0 \Rightarrow j_{2}=j_{3}=0, \quad j_{1}=\frac{1}{2} \Rightarrow j_{2}=j_{3} .
$$

The ordering of our the spins $j_{i} \in \frac{1}{2} \mathbb{Z}$ is such that the the first spin is related to the symplectic subalgebra $\mathfrak{s p}(2)$ while the two others are associated with the orthogonal one. This is a bit unfortunate but agrees with conventions in earlier literature. We shall use the label $[\Lambda]=\left[j_{1}, j_{2}, j_{3}\right]$ to denote finite dimensional irreducibles.

With these labels introduced we can now spell out the shortening conditions we have mentioned above. A representation $\left[j_{1}, j_{2}, j_{3}\right]$ is atypical provided the spins satisfy any one of the following conditions

$$
\begin{aligned}
& 2 j_{1}=-j_{2}-j_{3}, \\
& 2 j_{1}=j_{2}+j_{3}+2, \\
& 2 j_{1}= \pm\left(j_{2}-j_{3}\right)+1 .
\end{aligned}
$$

Otherwise the representation $\left[j_{1}, j_{2}, j_{3}\right]$ is typical. The eigenvalue of the quadratic Casimir element in the irreducible representation $[\Lambda]$ is given by

$$
\operatorname{Cas}_{\mathfrak{g}}(\Lambda)=-4 j_{1}\left(j_{1}-1\right)+2 j_{2}\left(j_{2}+1\right)+2 j_{3}\left(j_{3}+1\right) .
$$

If the spins satisfy one of the shortening conditions (A.3) the value of the quadratic Casimir element is a square, i.e. $\operatorname{Cas}_{\mathfrak{g}}(\Lambda)=l^{2}$ with $l \in \mathbb{N}$. The atypical weights $\Lambda=\left(j_{1}, j_{2}, j_{3}\right)$, i.e. those weights that satisfy one of the shortening conditions, can be divided into blocks $\beta_{l}$ that contain all those representations $\Lambda \in \beta_{l}$ for which $\operatorname{Cas}_{\mathfrak{g}}(\Lambda)=l^{2}$. The corresponding atypical labels can be listed explicitly [24],

$$
\begin{aligned}
& \beta_{0}=\left\{\Lambda_{0,0}=(0,0,0), \Lambda_{0, k}=\frac{1}{2}(k+1, k-1, k-1), k \geq 1\right\} \\
& \beta_{l}=\left\{\Lambda_{l, k}, k \in \mathbb{Z}\right\}
\end{aligned}
$$

where

$$
\Lambda_{l, k}=\left\{\begin{array}{llc}
\frac{1}{2}(-k+2,-k-l,-k+l) & \text { if } & k \leq-l \\
\frac{1}{2}(-k+1, k+l-1,-k+l-1) & \text { if } & -l+1 \leq k \leq 0 \\
\frac{1}{2}(k+1, k+l-1,-k+l-1) & \text { if } & 0 \leq k \leq l-1 \\
\frac{1}{2}(k+2, k+l, k-l) & \text { if } & l \leq k .
\end{array}\right.
$$

One sees easily, that the weights $\Lambda_{l,-k}$ for $l \geq 1$ may be obtained from $\Lambda_{l, k}$ by simply exchanging the second and the third Dynkin label. Furthermore, it is possible to distinguish the weights $\Lambda_{l, k}$ according to the atypicality condition (A.3) they obey. The only weight to fulfill the first condition is $\Lambda_{0,0}$. The weights belonging to the second condition are $\Lambda_{0, k}$ for $k \geq 1$ and $\Lambda_{l, \pm k}$ for $k \geq l$. Finally, those the satisfy the last atypicality relation are the $\Lambda_{l, \pm k}$ for $k<l$. In any case, each of the weights fulfills at most one of the shortening 
conditions. This means that all atypical representations of $\mathfrak{o s p}(4 \mid 2)$ possess the same degree of atypicality, i.e. they are all what mathematicians refer to as maximally atypical and physicists call $\frac{1}{2} \mathrm{BPS}$.

We can decompose all irreducible representations $\left[j_{1}, j_{2}, j_{3}\right]$ in terms of irreducible subrepresentations of the bosonic subalgebra $\mathfrak{g}_{\overline{0}}$. For typical representation one finds

$$
\begin{aligned}
{\left[j_{1}, j_{2}, j_{3}\right]_{g_{\overline{0}}} \cong } & \left(j_{1}, j_{2}, j_{3}\right) \bigoplus_{\alpha, \beta= \pm \frac{1}{2}}\left(j_{1}-\frac{1}{2}, j_{2}+\alpha, j_{3}+\beta\right) \\
& \bigoplus_{\alpha= \pm 1}\left[\left(j_{1}-1, j_{2}+\alpha, j_{3}\right) \oplus\left(j_{1}-1, j_{2}, j_{3}+\alpha\right)\right] \oplus 2\left(j_{1}-1, j_{2}, j_{3}\right) \\
& \oplus \bigoplus_{\alpha, \beta= \pm \frac{1}{2}}\left(j_{1}-\frac{3}{2}, j_{2}+\alpha, j_{3}+\beta\right) \oplus\left(j_{1}-2, j_{2}, j_{3}\right)
\end{aligned}
$$

There are a few special cases for which the decomposition is not generic. If $j_{1} \leq 2, j_{2} \leq 1$ or $j_{3} \leq 1$ then the above decomposition formula must be truncated at the point where one or more of the labels become negative. Moreover, there are two cases for which the multiplicity of the $\left(j_{1}-1, j_{2}, j_{3}\right)$ submodule has to be changed. If $j_{1}=1, j_{2}>0, j_{3}>0$ or $j_{1}>1, j_{2}=0, j_{3}>0$ or $j_{1}>1, j_{2}>0, j_{3}=0$, then this block will appear only once and if both $j_{2}$ and $j_{3}$ are null or $j_{1}=1$ and at least one between $j_{2}$ and $j_{3}$ is null, then it will not be present at all. From the decomposition into representations of the bosonic algebra we can determine the dimension of typical representations

$$
\operatorname{dim}\left[j_{1}, j_{2}, j_{3}\right]=16\left(2 j_{1}-1\right)\left(2 j_{2}+1\right)\left(2 j_{3}+1\right) .
$$

The decomposition (A.7) for $j_{1} \geq 1$, is valid for the indecomposable Kac modules that emerge when the spins $j_{i}$ satisfy one of the shortening conditions (A.3). These Kac modules are composites of irreducibles. More precisely, one finds

$$
\begin{array}{rlll}
K_{\Lambda_{0,2}}: & {\left[\Lambda_{0,2}\right] \longrightarrow\left[\Lambda_{0,0}\right] \oplus\left[\Lambda_{0,1}\right]} & & \\
K_{\Lambda_{0, k}}: & {\left[\Lambda_{0, k}\right] \longrightarrow\left[\Lambda_{0, k-1}\right]} & \text { for } \quad k \geq 3 \\
K_{\Lambda_{l, k}}: & {\left[\Lambda_{l, k}\right] \longrightarrow\left[\Lambda_{l, k-1}\right]} & \text { for } \quad k \geq 1 \\
K_{\Lambda_{l, k}}: & {\left[\Lambda_{l, k}\right] \longrightarrow\left[\Lambda_{l, k+1}\right]} & \text { for } \quad k \leq-1 .
\end{array}
$$

The arrows mean that fermionic generators can take us from the representation on the left to the one on the right but not vice versa. Put differently, the representation on the right hand side of the arrows is a subrepresentation of the Kac module. If we quotient the Kac module by this subrepresentation, the corresponding factor representation is the one on the left hand side. The representations with $j_{1}=\frac{1}{2}$ are somewhat special. In fact, when $j_{1}=\frac{1}{2}$, the Kac module is irreducible and we obtain

$$
\left.\Lambda_{l+1,2}\right|_{\mathfrak{g}_{\overline{0}}}=\left[\frac{1}{2}, \frac{l}{2}, \frac{l}{2}\right]_{\mathfrak{g}_{\overline{0}}} \cong\left(\frac{1}{2}, \frac{l}{2}, \frac{l}{2}\right) \oplus\left(0, \frac{l+1}{2}, \frac{l+1}{2}\right) \oplus\left(0, \frac{l-1}{2}, \frac{l-1}{2}\right) .
$$


From our description of the Kac modules it is possible to determine the dimensions of irreducible atypicals,

$$
\begin{aligned}
\operatorname{dim}\left[\Lambda_{0,0}\right] & =1, \quad \operatorname{dim}\left[\Lambda_{0,1}\right]=17, \quad \operatorname{dim}\left[\Lambda_{l, 0}\right]=4 l^{2}+2 & & \\
\operatorname{dim}\left[\Lambda_{0, k}\right] & =(2 k+1)\left[(2 k+1)^{2}-3\right] & & \text { for } \quad k \geq 2 \\
\operatorname{dim}\left[\Lambda_{l, k}\right] & =(2 k+1)\left[4\left(l^{2}-1\right)-(2 k+1)^{2}+7\right] & & \text { for } \quad k \leq l-1 \\
\operatorname{dim}\left[\Lambda_{l, k}\right] & =(2 k+3)\left[(2 k+3)^{2}-4\left(l^{2}-1\right)-7\right] & & \text { for } \quad k \geq l .
\end{aligned}
$$

We are finally prepared to describe the projective covers that feature so prominently in the construction of homogeneous vector bundles. While typical irreducibles $[\Lambda]$ coincide with their projective cover $\mathcal{P}_{\Lambda}=[\Lambda]$, the projective cover of an atypical representations is an indecomposable composite of atypicals. Its precise structure can be read off from the following diagrams

$$
\begin{aligned}
& \mathcal{P}_{\Lambda_{0,0}}: \Lambda_{0,0} \rightarrow \Lambda_{0,2} \rightarrow \Lambda_{0,0} \\
& \mathcal{P}_{\Lambda_{0,1}}: \Lambda_{0,1} \rightarrow \Lambda_{0,2} \rightarrow \Lambda_{0,1} \\
& \mathcal{P}_{\Lambda_{0,2}}: \Lambda_{0,2} \rightarrow \Lambda_{0,3} \oplus \Lambda_{0,1} \oplus \Lambda_{0,0} \rightarrow \Lambda_{0,2} \\
& \mathcal{P}_{\Lambda_{l, k}}: \Lambda_{l, k} \rightarrow \Lambda_{l, k+1} \oplus \Lambda_{l, k-1} \rightarrow \Lambda_{l, k} \quad \text { otherwise. }
\end{aligned}
$$

The meaning of the arrows was explained in our discussion of Kac modules above. Note that all the atypicals that appear in any given projective cover belong to the same block $\beta$. It is actually not possible to build indecomposables from representations within different blocks.

Before we conclude this brief overview over representations of the Lie superalgebra $\mathfrak{o s p}(4 \mid 2)$ we want to spell out a few tensor product decompositions between irreducible atypicals. These are used in our discussion of the low lying spectrum in the $\mathfrak{o s p}(4 \mid 2)$ Gross-Neveu model.

$$
\begin{aligned}
\Lambda_{0,1} \otimes \Lambda_{0,1}= & \Lambda_{0,0}+2 \Lambda_{0,1}+\Lambda_{0,2}+\Lambda_{2,-1}+2 \Lambda_{2,0}+\Lambda_{2,1}+[2,0,0] \\
\Lambda_{0,1} \odot \Lambda_{0,1}= & \Lambda_{0,0}+\Lambda_{2,-1}+2 \Lambda_{2,0}+\Lambda_{2,1}+[2,0,0] \\
\Lambda_{0,1} \otimes \Lambda_{0,2}= & \Lambda_{0,0}+\Lambda_{0,1}+3 \Lambda_{0,2}+\Lambda_{0,3}+ \\
& +[1,1,1]+\left[\frac{3}{2}, \frac{1}{2}, \frac{3}{2}\right]+\left[\frac{3}{2}, \frac{3}{2}, \frac{1}{2}\right]+[2,0,1]+[2,1,0]+\left[\frac{5}{2}, \frac{1}{2}, \frac{1}{2}\right] \\
\Lambda_{0,2} \otimes \Lambda_{0,2}= & 2 \Lambda_{0,0}+4 \Lambda_{0,1}+4 \Lambda_{0,2}+4 \Lambda_{0,3}+\Lambda_{0,4}+ \\
& +\Lambda_{2,-2}+3 \Lambda_{2,-1}+4 \Lambda_{2,0}+3 \Lambda_{2,1}+\Lambda_{2,2}+ \\
& +\Lambda_{4,-1}+2 \Lambda_{4,0}+\Lambda_{4,1}+ \\
& +[1,0,2]+2[1,1,1]+[1,2,0]+2\left[\frac{3}{2}, \frac{1}{2}, \frac{3}{2}\right]+2\left[\frac{3}{2}, \frac{3}{2}, \frac{1}{2}\right]+2\left[\frac{3}{2}, \frac{3}{2}, \frac{3}{2}\right]+ \\
& +2[2,0,0]+2[2,0,1]+2[2,1,0]+[2,1,2]+[2,2,1]+ \\
& +2\left[\frac{5}{2}, \frac{1}{2}, \frac{1}{2}\right]+2\left[\frac{5}{2}, \frac{1}{2}, \frac{3}{2}\right]+2\left[\frac{5}{2}, \frac{3}{2}, \frac{1}{2}\right]+[3,0,0]+[3,0,1]+[3,1,0]+[3,1,1]
\end{aligned}
$$

$\Lambda_{1,0} \otimes \Lambda_{1,0}=\Lambda_{0,0}+\Lambda_{0,1}+\Lambda_{2,0}$

$\Lambda_{2,0} \otimes \Lambda_{2,0}=\Lambda_{0,0}+\Lambda_{0,1}+\Lambda_{2,-1}+2 \Lambda_{2,0}+\Lambda_{2,1}+\Lambda_{4,0}+[1,1,1]$. 
The + on the right hand side requires a short comment. As we have stated above, atypical irreducibles can be combined to form larger indecomposables. This happens for many of the atypical representations that appear in the above tensor product decompositions. Hence, many of the atypicals are not direct summands. This is why we did not use $\oplus$. On the other hand, the sum is direct for all projective modules, i.e. for typicals and projective covers of atypicals. The symbol $\odot$ is used to denote the symmetric part of the tensor product.

\section{B Representation theory of $\mathfrak{o s p}(3 \mid 2)$}

In this appendix we provide some background material on the Lie superalgebra $\mathfrak{o s p}(3 \mid 2)$ and its finite dimensional representations. The basic definition of $\mathfrak{o s p}(3 \mid 2)$ resembles the definition (A.1) we gave for $\mathfrak{o s p}(4 \mid 2)$ only that now $A$ is a $3 \times 3$ matrix and $B$ is rectangular of size $3 \times 2$. In the case of $\mathfrak{h}=\mathfrak{o s p}(3 \mid 2)$, the bosonic subalgebra is $\mathfrak{h}_{\overline{0}}=\mathfrak{s o}(3) \oplus \mathfrak{s p}(2)$. Since $\mathfrak{h}_{\overline{0}}$ has rank two, highest weights are labeled by two numbers $\lambda=(q, p)$. In our conventions, the $\mathfrak{s o}(3)$ spin $p$ runs over non-negative integers while $q$ is a non-negative half-integer. Note that once again, the order of the two labels is a bit unfortunate. As in the case of $\mathfrak{o s p}(4 \mid 2)$, there is an additional constraint on the weights $(q, p)$ that must be satisfied in order for $(q, p)$ to label a representation of $\mathfrak{o s p}(3 \mid 2)$, namely

$$
q=0 \Rightarrow p=0 .
$$

Once more we shall use the bracket notation $[\lambda]=[q, p]$ to denote the associated irreducible representation of $\mathfrak{o s p}(3 \mid 2)$. The representation $[q, p]$ is typical (long) unless the labels $q, p$ satisfy one of the following two shortening conditions

$$
p+2 q=0 \quad p-2 q+1=0 .
$$

These conditions are mutually exclusive. While the first one is only satisfied for the trivial representation $q=p=0$, the latter singles out a one parameter family of (maximally) atypical (or $\frac{1}{2} \mathrm{BPS}$ ) representations.

The eigenvalue of the quadratic Casimir element in an irreducible representation $[\lambda]=$ $[q, p]$ is given by

$$
\operatorname{Cas}_{\mathfrak{h}}([q, p])=(p+2 q)(p-2 q+1) .
$$

In particular, we conclude that the quadratic Casimir element vanishes for all atypical representations of $\mathfrak{o s p}(3 \mid 2)$. This suggests that all atypicals belong to one and the same block, which is indeed the case. Representations in this unique block are given by

$$
\lambda_{0}=[0,0], \quad \lambda_{q}=[q, 2 q-1] .
$$

Let us also mention in passing that the Lie superalgebra $\mathfrak{o s p}(3 \mid 2)$ possesses a fourth order Casimir element whose eigenvalues are given by

$$
\operatorname{Cas}_{\mathfrak{h}}^{(4)}(\lambda)=\frac{1}{4} \operatorname{Cas}_{\mathfrak{h}}(\lambda)[3 p(3 p+1)+2(q+1)(2 q-3)] .
$$

The fourth order Casimir element does not show up in the 1-loop anomalous dimensions but could enter starting from 2 loops. 
As in the case of $\mathfrak{o s p}(4 \mid 2)$ it is useful to know how the irreducible representations decompose with respect to the bosonic subalgebra. For typical representations, this decomposition is given by

$$
[q, p]_{\mathfrak{h}_{\overline{0}}} \cong(q, p) \oplus \bigoplus_{\alpha=0, \pm 1}\left[\left(q-\frac{1}{2}, p+\alpha\right) \oplus(q-1, p+\alpha)\right] \oplus\left(q-\frac{3}{2}, p\right) .
$$

Truncations are present whenever one or both labels on the right hand side become negative. When $q=\frac{1}{2}$ or $p=0$ the term $\left(q-\frac{1}{2}, p\right)$ does not appear. For the adjoint representation the decomposition reads

$$
[1,0]_{\mathfrak{h}_{\overline{0}}} \cong(1,0) \oplus\left(\frac{1}{2}, 1\right) \oplus(0,1) .
$$

Note that in the case of $\mathfrak{o s p}(3 \mid 2)$ the adjoint representation is typical. Atypical representations with $q \geq 1$ possess the following decomposition

$$
\left[\lambda_{q}\right]_{\mathfrak{h}_{\overline{0}}} \cong(q, 2 q-1) \oplus\left(q-\frac{1}{2}, 2 q-1\right) \oplus\left(q-\frac{1}{2}, 2 q\right) \oplus(q-1,2 q) .
$$

The atypical trivial representation $\lambda_{0}$ and the fundamental $\lambda_{\frac{1}{2}}$ are special. While the decomposition of $\lambda_{0}$ is trivial, the fundamental representation gives

$$
\left[\lambda_{\frac{1}{2}}\right]_{\mathfrak{h} \overline{0}} \cong\left(\frac{1}{2}, 0\right) \oplus(0,1) .
$$

For completeness we also state the dimension of the these representations. In the case of typical long multiplets we have

$$
\operatorname{dim}([q, p])=4(2 p+1)(4 p-1)
$$

while the dimension of atypicals is given by

$$
\begin{aligned}
& \operatorname{dim}\left[\lambda_{0}\right]=1 \quad \operatorname{dim}\left[\lambda_{\frac{1}{2}}\right]=5 \\
& \operatorname{dim}\left[\lambda_{q}\right]=-2+32 q^{2} .
\end{aligned}
$$

As for any Lie superalgebra, atypical representations can be combined into larger indecomposables. For our analysis, the projective covers of atypicals are of particular importance. Their structure is given by

$$
\begin{aligned}
& \mathcal{P}_{\lambda_{0}}: \lambda_{0} \rightarrow \lambda_{1} \rightarrow \lambda_{0} \\
& \mathcal{P}_{\lambda_{\frac{1}{2}}}: \lambda_{\frac{1}{2}} \rightarrow \lambda_{1} \rightarrow \lambda_{\frac{1}{2}} \\
& \mathcal{P}_{\lambda_{1}}: \lambda_{1} \rightarrow \lambda_{\frac{3}{2}} \oplus \lambda_{\frac{1}{2}} \oplus \lambda_{0} \rightarrow \lambda_{1} \\
& \mathcal{P}_{\lambda_{q}}: \lambda_{q} \rightarrow \lambda_{q+\frac{1}{2}} \oplus \lambda_{q-\frac{1}{2}} \rightarrow \lambda_{q} \quad \text { otherwise. }
\end{aligned}
$$

The meaning of the arrows was explained in appendix A. The structure we display here is consistent with the fact that all atypical irreducibles $\lambda_{q}$ of $\mathfrak{o s p}(3 \mid 2)$ belong to the same block. 
In our construction of coset vertex operators (2.8), and in particular in the analysis of the tail factors, we need some input about tensor products of $\mathfrak{o s p}(3 \mid 2)$ representations. The first few powers of the fundamental representation $\lambda_{\frac{1}{2}}$ are given by

$$
\begin{aligned}
& \lambda_{\frac{1}{2}}^{\otimes 2}=[1,0]+\left[\frac{1}{2}, 1\right]+\lambda_{0} \\
& \lambda_{\frac{1}{2}}^{\odot 2}=\left[\frac{1}{2}, 1\right]+\lambda_{0} \\
& \lambda_{\frac{1}{2}}^{\odot 3}=\left[\frac{1}{2}, 2\right]+\lambda_{\frac{1}{2}} .
\end{aligned}
$$

Here, we use the symbol $\odot$ to denote the graded symmetric part of the tensor product. The formulas we displayed are relevant e.g. for products such as $j \partial j, j^{2}$ and $j^{3}$, respectively. Let us also list a few additional tensor products of low dimensional representations,

$$
\begin{aligned}
{[1,0] \otimes \lambda_{\frac{1}{2}} } & =\left[\frac{3}{2}, 0\right]+2 \lambda_{\frac{1}{2}}+\lambda_{1} \\
{\left[\frac{1}{2}, 1\right] \otimes \lambda_{\frac{1}{2}} } & =\left[\frac{1}{2}, 2\right]+2 \lambda_{\frac{1}{2}}+\lambda_{1} \\
{\left[\frac{1}{2}, 1\right] \otimes\left[\frac{1}{2}, 1\right] } & =[1,2]+[1,0]+\left[\frac{1}{2}, 3\right]+\left[\frac{1}{2}, 1\right]+2 \lambda_{0}+\lambda_{1} \\
{\left[\frac{1}{2}, 2\right] \otimes \lambda_{\frac{1}{2}} } & =[1,2]+\left[\frac{1}{2}, 3\right]+\left[\frac{1}{2}, 1\right] \\
{\left[\frac{1}{2}, 2\right] \otimes\left[\frac{1}{2}, 1\right] } & =[1,3]+[1,2]+\left[\frac{1}{2}, 4\right]+\left[\frac{1}{2}, 2\right]+2 \lambda_{\frac{1}{2}}+\lambda_{1} \\
{\left[\frac{1}{2}, 2\right] \otimes\left[\frac{1}{2}, 2\right] } & =[1,4]+[1,3]+[1,2]+[1,0]+\left[\frac{1}{2}, 5\right]+\left[\frac{1}{2}, 3\right] \\
& +\left[\frac{1}{2}, 2\right]+\left[\frac{1}{2}, 1\right]+2 \lambda_{0}+\lambda_{1} \\
{\left[\frac{1}{2}, 1\right] \otimes[1,0] } & =\left[\frac{3}{2}, 1\right]+[1,0]+[1,2]+\left[\frac{1}{2}, 1\right] \\
{\left[\frac{1}{2}, 2\right] \otimes[1,0] } & =[1,3]+\left[\frac{1}{2}, 2\right]+\lambda_{0}+\lambda_{\frac{1}{2}}+2 \lambda_{1}+\lambda_{\frac{3}{2}} \\
{[1,0] \otimes[1,0] } & =[2,0]+\left[\frac{3}{2}, 1\right]+[1,0]+\left[\frac{1}{2}, 1\right]+2 \lambda_{0}+\lambda_{1} .
\end{aligned}
$$

These are useful in order to carry the construction of vertex operators to higher gradient operators. Note that while it is not relevant for our discussion, the atypical representations in (B.18) always combine into projectives, while all other sums are direkt.

\section{Restriction of $\mathfrak{o s p}(4 \mid 2)$ representations to $\mathfrak{o s p}(3 \mid 2)$}

As we explained in section 2.3, a key ingredient in constructing vertex operators on coset superspaces is the decomposition (2.9) of sections in homogeneous vector bundles into multiplets of the symmetry. According to the central formula, the multiplicity $n_{\Lambda \lambda}$ of a $\mathfrak{g}$ 
multiplet $\Lambda$ in a bundle $\Gamma_{\lambda}$ is given by eqn (2.11). It implies that $n_{\Lambda \lambda}$ can be computed through the decomposition

$$
\left.\mathcal{P}_{\Lambda}\right|_{\mathfrak{h}}=\bigoplus_{\lambda} n_{\Lambda \lambda} \mathcal{P}_{\lambda}=\bigoplus_{\lambda}\left[\left.\mathcal{P}_{\Lambda}\right|_{\mathfrak{h}}: \mathcal{P}_{\lambda}\right] \mathcal{P}_{\lambda}
$$

Given what we know about the projective covers of both $\mathfrak{o s p}(4 \mid 2)$ and $\mathfrak{o s p}(3 \mid 2)$ it is not too difficult to work out the multiplicities $n_{\Lambda \lambda}$. We only need the results for atypical labels $\Lambda=\Lambda_{l, k}$. For representations $\Lambda_{0, k}$ in the block of the trivial representation one finds

$$
\begin{aligned}
& \mathcal{P}_{\left.\Lambda_{0,0}\right|_{\text {osp }(3 \mid 2)}}=\mathcal{P}_{\lambda_{0}} \oplus\left[\frac{3}{2}, 0\right] \oplus\left[\frac{3}{2}, 1\right] \\
& \mathcal{P}_{\left.\Lambda_{0,1}\right|_{\text {osp }(3 \mid 2)}}=\mathcal{P}_{\lambda_{\frac{1}{2}}} \oplus\left[\frac{3}{2}, 0\right] \oplus\left[\frac{3}{2}, 1\right] \\
& \mathcal{P}_{\left.\Lambda_{0, k}\right|_{\text {osp }(3 \mid 2)}}=\mathcal{P}_{\lambda_{\frac{k}{2}}} \oplus 2 \bigoplus_{n=0}^{k-1}\left[\frac{k+1}{2}, n\right] \oplus \bigoplus_{n=0}^{k}\left[\frac{k+2}{2}, n\right] \oplus \bigoplus_{n=0}^{k-2}\left[\frac{k}{2}, n\right], \text { for all } k \geq 2 .
\end{aligned}
$$

Similarly one can decompose the projective covers of the symmetric traceless tensor representations $\Lambda_{l, 0}$,

$$
\begin{aligned}
& \left.\mathcal{P}_{\Lambda_{1,0}}\right|_{\text {osp }(3 \mid 2)}=\mathcal{P}_{\lambda_{0}} \oplus \mathcal{P}_{\lambda_{\frac{1}{2}}} \oplus 2\left[\frac{3}{2}, 1\right] \\
& \mathcal{P}_{\left.\Lambda_{2,0}\right|_{\text {ofp }(3 \mid 2)}}=\mathcal{P}_{\lambda_{0}} \oplus \mathcal{P}_{\lambda_{\frac{1}{2}}} \oplus 2\left[\frac{1}{2}, 1\right] \\
& \left.\mathcal{P}_{\Lambda_{l, 0}}\right|_{\text {osp }(3 \mid 2)}=\mathcal{P}_{\lambda_{0}} \oplus \mathcal{P}_{\lambda_{\frac{1}{2}}} \oplus 2 \bigoplus_{n=1}^{l-1}\left[\frac{1}{2}, n\right] \oplus 2 \bigoplus_{n=2}^{l-1}[1, n], \quad \text { when } \quad l \geq 2 .
\end{aligned}
$$

Finally, generic projective covers possess the following decomposition into projectives of $\mathfrak{o s p}(3 \mid 2)$,

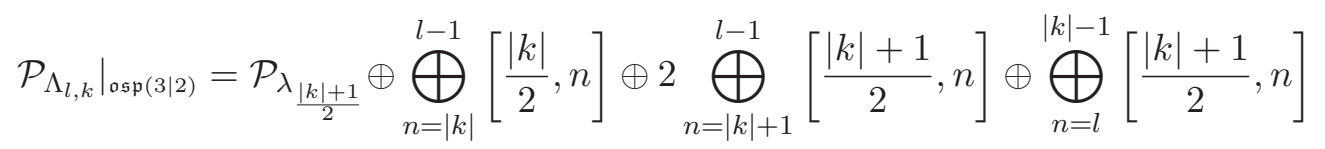

$$
\begin{aligned}
& \oplus 2 \bigoplus_{n=l}^{|k|}\left[\frac{|k|+2}{2}, n\right] \oplus \bigoplus_{n=|k|+2}^{l-1}\left[\frac{|k|+2}{2}, n\right] \oplus \bigoplus_{n=l}^{|k|+1}\left[\frac{|k|+3}{2}, n\right] .
\end{aligned}
$$

This last formula holds whenever $l \geq 1$ and $|k| \geq 1$. Formulas (C.1)-(C.3) provide the main input for the construction of vertex operators in section 3.2. Let us note that in these formulas all sums are direct since the restriction of projective modules is a direct sum of projectives and projectives cannot appear as pieces of larger indecomposibles.

In order to derive these decomposition formulas one starts from the following decomposition formula for representations of the bosonic subalgebra $\mathfrak{g}_{\overline{0}}$ into representations of $\mathfrak{h}_{\overline{0}}$,

$$
\left(j_{1}, j_{2}, j_{3}\right)_{\mathfrak{h}_{\overline{0}}} \cong \bigoplus_{p=\left|j_{2}-j_{3}\right|}^{j_{2}+j_{3}}\left(j_{1}, p\right)
$$


In a second step these decomposition formulas are exploited to determine how atypical irreducibles of $\mathfrak{o s p}(4 \mid 2)$ decompose upon restriction to $\mathfrak{o s p}(3 \mid 2)$. The results read,

$$
\begin{aligned}
& \left.\Lambda_{0,0}\right|_{\operatorname{osp}(3 \mid 2)}=\lambda_{0} \\
& \left.\Lambda_{0, k}\right|_{\text {osp }(3 \mid 2)}=\lambda_{\frac{l}{2}} \oplus \bigoplus_{n=0}^{k-1}\left[\frac{k+1}{2}, n\right], \quad l>0 \\
& \left.\Lambda_{l, 0}\right|_{\text {osp }(3 \mid 2)}=\bigoplus_{n=0}^{l-1}\left[\frac{1}{2}, n\right] \oplus \lambda_{0}, \quad l>0 \\
& \left.\Lambda_{l, k}\right|_{\mathfrak{o s p}(3 \mid 2)}=\bigoplus_{n=|k|}^{l-1}\left[\frac{|k|+1}{2}, n\right], \quad 0<|k| \leq l-1 \\
& \left.\Lambda_{l, k}\right|_{\mathfrak{o s p}(3 \mid 2)}=\bigoplus_{n=l}^{|k|}\left[\frac{|k|}{2}+1, n\right] \oplus \lambda_{\frac{|k|+1}{2}}, \quad 0<l \leq|k| .
\end{aligned}
$$

Since we know how projective covers are built from atypicals, it is now straightforward to verify the decomposition formulas (C.1)-(C.3).

Open Access. This article is distributed under the terms of the Creative Commons Attribution License (CC-BY 4.0), which permits any use, distribution and reproduction in any medium, provided the original author(s) and source are credited.

\section{References}

[1] A.M. Polyakov, Interaction of Goldstone Particles in Two-Dimensions. Applications to Ferromagnets and Massive Yang-Mills Fields, Phys. Lett. B 59 (1975) 79 [inSPIRE].

[2] E. Brézin and J. Zinn-Justin, Renormalization of the nonlinear $\sigma$-model in $2+\epsilon$ dimensions. Application to the Heisenberg ferromagnets, Phys. Rev. Lett. 36 (1976) 691 [INSPIRE].

[3] D.H. Friedan, Nonlinear Models in $2+\epsilon$ Dimensions, Annals Phys. 163 (1985) 318 [INSPIRE].

[4] A. D'Adda, M. Lüscher and P. Di Vecchia, A 1/n Expandable Series of Nonlinear $\sigma$-models with Instantons, Nucl. Phys. B 146 (1978) 63 [INSPIRE].

[5] I. Bena, J. Polchinski and R. Roiban, Hidden symmetries of the Ad $S_{5} \times S^{5}$ superstring, Phys. Rev. D 69 (2004) 046002 [hep-th/0305116] [inSPIRE].

[6] S.R. Coleman, The Quantum sine-Gordon Equation as the Massive Thirring Model, Phys. Rev. D 11 (1975) 2088 [INSPIRE].

[7] S. Mandelstam, Soliton Operators for the Quantized sine-Gordon Equation, Phys. Rev. D 11 (1975) 3026 [INSPIRE].

[8] E. Witten, Phases of $N=2$ theories in two-dimensions, Nucl. Phys. B 403 (1993) 159 [hep-th/9301042] [INSPIRE].

[9] C. Candu, V. Mitev and V. Schomerus, Spectra of Coset $\sigma$-models, Nucl. Phys. B 877 (2013) 900 [arXiv:1308.5981] [InSPIRE]. 
[10] C. Candu and H. Saleur, A lattice approach to the conformal $\operatorname{OSp}(2 S+2 \mid 2 S)$ supercoset $\sigma$-model. Part I: Algebraic structures in the spin chain. The Brauer algebra,

Nucl. Phys. B 808 (2009) 441 [arXiv:0801.0430] [INSPIRE].

[11] C. Candu and H. Saleur, A lattice approach to the conformal $\operatorname{OSp}(2 S+2 \mid 2 S)$ supercoset $\sigma$-model. Part II: The boundary spectrum, Nucl. Phys. B 808 (2009) 487 [arXiv:0801.0444] [INSPIRE].

[12] V. Mitev, T. Quella and V. Schomerus, Principal Chiral Model on Superspheres, JHEP 11 (2008) 086 [arXiv:0809.1046] [INSPIRE].

[13] C. Candu, V. Mitev and V. Schomerus, Anomalous Dimensions in Deformed WZW Models on Supergroups, JHEP 03 (2013) 003 [arXiv:1211.2238] [INSPIRE].

[14] A.M. Polyakov, Supermagnets and $\sigma$-models, hep-th/0512310 [INSPIRE].

[15] T. Quella, V. Schomerus and T. Creutzig, Boundary Spectra in Superspace $\sigma$-models, JHEP 10 (2008) 024 [arXiv:0712.3549] [INSPIRE].

[16] C. Candu, V. Mitev, T. Quella, H. Saleur and V. Schomerus, The $\sigma$-model on Complex Projective Superspaces, JHEP 02 (2010) 015 [arXiv:0908.0878] [InSPIRE].

[17] M. Bershadsky, S. Zhukov and A. Vaintrob, $P S L(n \mid n) \sigma$-model as a conformal field theory, Nucl. Phys. B 559 (1999) 205 [hep-th/9902180] [inSPIRE].

[18] N. Berkovits, C. Vafa and E. Witten, Conformal field theory of AdS background with Ramond-Ramond flux, JHEP 03 (1999) 018 [hep-th/9902098] [INSPIRE].

[19] N. Read and H. Saleur, Exact spectra of conformal supersymmetric nonlinear $\sigma$-models in two-dimensions, Nucl. Phys. B 613 (2001) 409 [hep-th/0106124] [INSPIRE].

[20] D. Kagan and C.A.S. Young, Conformal $\sigma$-models on supercoset targets, Nucl. Phys. B 745 (2006) 109 [hep-th/0512250] [INSPIRE].

[21] A. Babichenko, Conformal invariance and quantum integrability of $\sigma$-models on symmetric superspaces, Phys. Lett. B 648 (2007) 254 [hep-th/0611214] [INSPIRE].

[22] C. Candu, Discrétisation des modèles sigma invariants conformes sur des supersphères et superespaces projectifs, Ph.D. Thesis, Université Pierre et Marie Curie - Paris VI, Paris, France (2008).

[23] R. Bott, Homogeneous vector bundles, Ann. Math. 66 (1957) 203.

[24] J. Germoni, Indecomposable representations of osp $(3 \mid 2), d(2,1 ; \alpha)$ and $g(3)$, Bol. Acad. Nac. Cienc. (Córdoba) 65 (2000) 147.

[25] T. Quella and V. Schomerus, Superspace conformal field theory, J. Phys. A 46 (2013) 494010 [arXiv:1307.7724] [InSPIRE].

[26] K. Pilch and A.N. Schellekens, Formulae for the Eigenvalues of the Laplacian on Tensor Harmonics on Symmetric Coset Spaces, J. Math. Phys. 25 (1984) 3455 [inSPIRE].

[27] V.E. Kravtsov, I.V. Lerner and V.I. Yudson, Anomalous Dimensions of High Gradient Operators in the Extended Nonlinear $\sigma$ Model and Distribution of Mesoscopic Fluctuations, Phys. Lett. A 134 (1989) 245 [inSPIRE].

[28] F. Wegner, Anomalous dimensions of high-gradient operators in the n-vector model in $2+\epsilon$ dimensions, Z. Phys. B 78 (1990) 33. 
[29] F. Wegner, Anomalous dimensions of high gradient operators in the unitary matrix model, Nucl. Phys. B 354 (1991) 441 [inSPIRE].

[30] H. Mall and F. Wegner, Anomalous dimensions of high gradient operators in the orthogonal matrix model, Nucl. Phys. B 393 (1993) 495 [InSPIRE].

[31] A.N. Vasiliev and A.S. Stepanenko, A method of calculating the critical dimensions of composite operators in the massless nonlinear $\sigma$-model, Theor. Math. Phys. 94 (1993) 471 [INSPIRE].

[32] K. Lang and W. Rühl, Critical nonlinear $O(N) \sigma$-models at $2<d<4$ : The degeneracy of quasiprimary fields and it resolution, Z. Phys. C 61 (1994) 495 [INSPIRE].

[33] S.E. Derkachov, S.K. Kehrein and A.N. Manashov, High-gradient operators in the N-vector model, Nucl. Phys. B 493 (1997) 660 [inSPIRE].

[34] S. Ryu, C. Mudry, A.W.W. Ludwig and A. Furusaki, High-gradient operators in perturbed Wess-Zumino-Witten field theories in two dimensions, Nucl. Phys. B 839 (2010) 341 [arXiv: 1002.0118] [INSPIRE].

[35] G.E. Castilla and S. Chakravarty, Is the phase transition in the Heisenberg model described by the (2+epsilon) expansion of the nonlinear $\sigma$-model?, Nucl. Phys. B 485 (1997) 613 [cond-mat/9605088] [INSPIRE].

[36] E. Witten, Nonabelian Bosonization in Two-Dimensions, Commun. Math. Phys. 92 (1984) 455 [InSPIRE].

[37] J. Van der Jeugt, Irreducible representations of the exceptional lie superalgebras $D(2,1 \alpha)$, J. Math. Phys. 26 (1985) 913 [INSPIRE].

[38] J. Van der Jeugt, Finite and infinite-dimensional representations of the orthosymplectic superalgebra OSP(3,2), J. Math. Phys. 25 (1984) 3334 [InSPIRE].

[39] C. Candu, T. Creutzig, V. Mitev and V. Schomerus, Cohomological Reduction of $\sigma$-models, JHEP 05 (2010) 047 [arXiv: 1001.1344] [INSPIRE].

[40] F. Wegner, Anomalous Dimensions for the Nonlinear $\sigma$ Model in $(2+\epsilon)$-dimensions. 2, Nucl. Phys. B 280 (1987) 210 [InSPIRE].

[41] F. Wegner, Anomalous Dimensions for the Nonlinear $\sigma$ Model in $(2+\epsilon)$-dimensions. 1, Nucl. Phys. B 280 (1987) 193 [inSPIRE].

[42] C. Candu and V. Schomerus, Exactly marginal parafermions, Phys. Rev. D 84 (2011) 051704 [arXiv: 1104.5028] [INSPIRE]. 